\title{
BULK FLOWS AND COSMIC MICROWAVE BACKGROUND DIPOLE ANISOTROPY IN COSMOLOGICAL VOID MODELS
}

\author{
KeNJI TOMITA \\ Yukawa Institute for Theoretical Physics, Kyoto University, Kyoto 606-8502, Japan; tomita@yukawa.kyoto-u.ac.jp \\ Received 1999 June 23; accepted 1999 September 7
}

\begin{abstract}
The observational behavior of spherically symmetric inhomogeneous cosmological models is studied, which consist of inner and outer homogeneous regions connected by a shell or an intermediate selfsimilar region. It is assumed that the present matter density parameter in the inner region is smaller than that in the outer region, and the present Hubble parameter in the inner region is larger than that in the outer region. Then, galaxies in the inner voidlike region can be seen to have a bulk motion relative to matter in the outer region, when we observe them at a point $\mathrm{O}$ deviated from the center $\mathrm{C}$ of the inner region. Their velocity $v_{p}$ in the CD direction is equal to the difference of two Hubble parameters multiplied by the distance between $\mathrm{C}$ and $\mathrm{O}$. It is also found that the velocity $v_{d}$ corresponding to cosmic microwave background dipole anisotropy observed at $\mathrm{O}$ is by a factor of $\approx 10$ small compared with $v_{p}$. This behavior of $v_{d}$ and $v_{p}$ may explain the puzzling situation of the cosmic flow of cluster galaxies, when the radius of the inner region and the distance CD are about 200 and $40 h^{-1} \mathrm{Mpc}$, respectively $\left(H_{0}=100 h^{-1} \mathrm{~km} \mathrm{~s}^{-1} \mathrm{Mpc}^{-1}\right)$, and when the gaps of density and Hubble parameters are $\approx 0.5$ and $18 \%$, respectively.

Subject headings: cosmic microwave background - cosmology: theory -

large-scale structure of universe
\end{abstract}

\section{INTRODUCTION}

The dipole moment in the cosmic microwave background (CMB) radiation is thought to come mainly from the Doppler shift due to the motion of the Local Group (LG), relative to the cosmic homogeneous expansion. As the main gravitational source which brings the velocity vector of $L G$, the existence of the Great Attractor (GA) was found by Lynden-Bell et al. (1988) and Dressler et al. (1987). It has the position at a redshift of $4300 \mathrm{~km} \mathrm{~s}^{-1}$. On the other hand, the motion of LG in the inertial frame consisting of many clusters on larger scales was studied observationally by several groups: a bulk flow of $\sim 700 \mathrm{~km} \mathrm{~s}^{-1}$ was found by Lauer \& Postman (1994), Postman \& Lauer (1995), and Colless (1995) as the motion of the Abell cluster inertial frame relative to the LG in the region with redshift greater than $15,000 \mathrm{~km} \mathrm{~s}^{-1}$, but in the other approach a different result was derived by Giovanelli et al. (1998), Dale, Giovanelli, \& Haynes (1999), and Riess et al. (1997) in the regions with similar redshifts. Lauer \& Postman's (1994) and Postman \& Lauer's (1995) work is based on the assumption that the brightest cluster galaxies as standard candles and the Hoessel relation can be used, but at present these assumptions are regarded as questionable or unreliable.

Independently of these works, the motion of cluster frames relative to the CMB was measured by Hudson et al. (1999, 1997) and Willick (1999) with the global Hubble formula using the Tully-Fisher distances of clusters and their redshifts with respect to the CMB, and the flow velocity vector was derived in the region with about $150 \mathrm{~h}^{-1}$ Mpc $\left(H_{0}=100 h^{-1} \mathrm{~km} \mathrm{~s}^{-1} \mathrm{Mpc}^{-1}\right)$. The remarkable and puzzling properties of these flows are that the flow velocity reaches a large value of $\sim 700 \mathrm{~km} \mathrm{~s}^{-1}$ on a large scale, while the dipole velocity (not due to the GA) determined in the form of CMB dipole anisotropy seems to be much smaller compared with the above flow velocity.

If the observed large-scale matter motion is caused by the attraction from an overdensity region containing super- clusters, the corresponding velocity must be as large as the large-scale flow velocity and it must be reflected in the form of CMB dipole anisotropy. If this motion is caused in the spherical voidlike region, however, the situation is different, because CMB dipole anisotropy near the center can be relatively small in spite of the large-scale flow, as was shown in our previous paper (Tomita 1996). In our previous paper, an inhomogeneous model on superhorizon scale was considered to explain the number evolution of QSOs (Tomita 1995), but the relative smallness of the dipole anisotropy can be found independently of the scale of inhomogeneities. The local void region was studied independently by Zehavi et al. (1998) as the local Hubble bubble, which has the scale $\sim 70 h^{-1} \mathrm{Mpc}$ and is bordered by dense walls. They analyzed the statistical relation between the distances and the local Hubble constants derived from the data of supernovae $(\mathrm{SNe}) \mathrm{Ia}$, and found the existence of a void region with a local Hubble constant larger than the global Hubble constant. The relation to the SN Ia data on larger scales will be discussed from our standpoint in a subsequent paper.

In the present paper, we consider more realistic inhomogeneous models on the subhorizon scale, corresponding to matter flows of $\sim 150 h^{-1} \mathrm{Mpc}$, which may be associated with large-scale structures or excess powers observed by Broadhurst et al. (1990), Landy et al. (1996), and Einasto et al. (1997). In $\S 2$, we treat a spherically symmetric inhomogeneous model, which consists of inner and outer homogeneous regions connected by a shell, as a singular layer and study the behavior of large-scale motions caused in the inner region, where the present inner density parameter is smaller than the present outer density parameter and the present Hubble parameter in the inner region is larger than that in the outer region (a bulk motion in the voidlike region was discussed also by Nakao et al. 1995). In this section, we treat the single-shell case. The double-shell case and a model with an intermediate self-similar region are treated in Appendixes A and B, respectively. In $\S 3$, we 
consider light rays which are emitted at the last scattering surface and reach an observer situated at a point $O$ deviated from the center C, and CMB dipole and quadrupole anisotropies are analyzed. The peculiar velocity of the above large-scale motions and the velocity corresponding to the CMB dipole anisotropy are compared. In $\S 4$, a naive explanation about why CMB dipole anisotropy is small around the center $\mathrm{C}$ is shown. In $\S 5$, the consistency of the present models with several recent observations of bulk flows is discussed, and in $\S 6$, concluding remarks are presented.

\section{INHOMOGENEOUS MODELS AND BULK MOTION}

In this section, we consider spherically symmetric inhomogeneous cosmological models which have two homogeneous regions connected with a spherical shell, as shown in Figure 1.

The line elements in the inner region $\mathrm{V}^{\mathrm{I}}$ and the outer region $\mathrm{V}^{\mathrm{II}}$ are described as

$$
\begin{aligned}
d s^{2} & =g_{\mu \nu}^{j}\left(d x^{j}\right)^{\mu}\left(d x^{j}\right)^{v} \\
& =-c^{2}\left(d t^{j}\right)^{2}+\left[a^{j}\left(t^{j}\right)\right]^{2}\left\{d\left(\chi^{j}\right)^{2}+\left[f^{j}\left(\chi^{j}\right)\right]^{2} d \Omega^{2}\right\},
\end{aligned}
$$

where $j\left(=\mathrm{I}\right.$ or II) represents the regions; $f^{j}\left(\chi^{j}\right)=\sin \chi^{j}, \chi^{j}$, and $\sinh \chi^{j}$ for $k^{j}=1,0,-1$, respectively; and $d \Omega^{2}=d \theta^{2}+\sin ^{2} \theta \varphi^{2}$. The shell is a timelike hypersurface $\Sigma$ given as $\chi^{\mathrm{I}}=\chi_{1}^{\mathrm{I}}$ and $\chi^{\mathrm{II}}=\chi_{1}^{\mathrm{II}}$.

\subsection{Cosmological Models}

The Einstein equations are divided into the equations in the two regions and the jump conditions at the shell. The general formulation of the jump condition at the singular surface was derived by Israel (1966) and the concrete expressions of conditions were derived by Maeda (1986) and

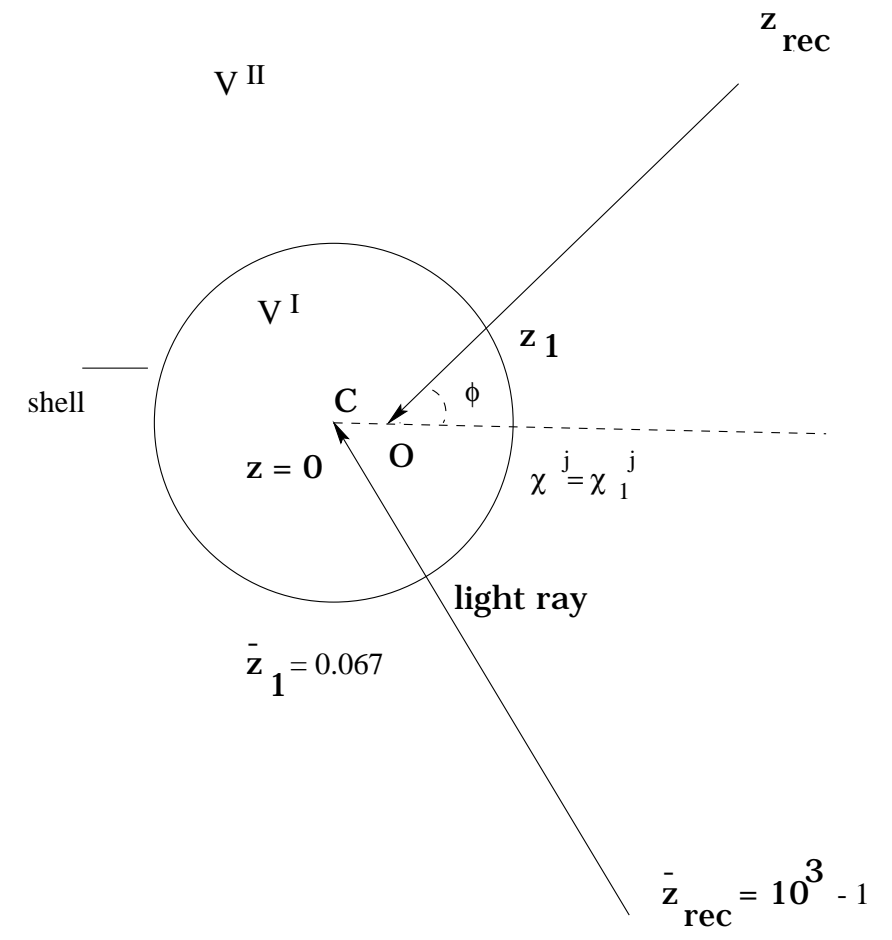

FIG. 1.- Model with a single shell. $z$ and $\bar{z}$ are the redshifts for observers at $\mathrm{O}$ and $\mathrm{C}$.

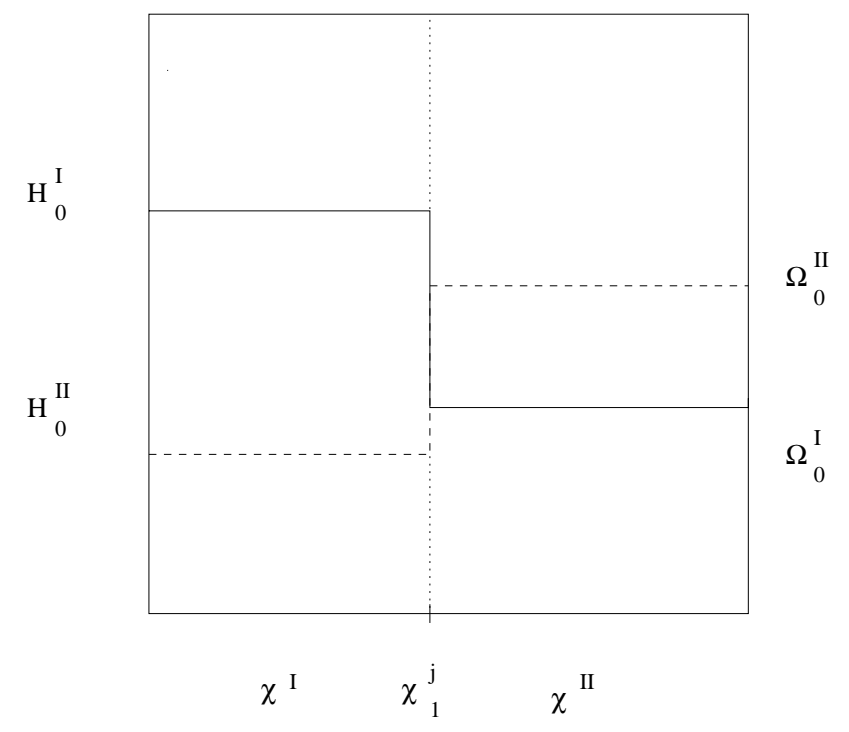

FIG. 2.- Schematic diagram of Hubble and density parameters. Solid and dotted lines denote $H_{0}$ and $\Omega_{0}$, respectively.

Sakai, Maeda, \& Sato (1993). Here the expressions by Sakai et al. are shown using the circumferential radius of the shell $R$, the velocity of the shell $v^{j}$, the Lorentz factor $\gamma^{j}$, and the Hubble expansion parameter $H^{j}$ in $V^{j}(j=\mathrm{I}$ and II), defined by

$$
\begin{gathered}
R \equiv a^{\mathrm{I}} f^{\mathrm{I}}=a^{\mathrm{I} f} f^{\mathrm{II}}, \quad v^{j} \equiv a_{j} \frac{d \chi^{j}}{d t^{j}}, \\
\gamma^{j} \equiv 1 / \sqrt{1-\left(\frac{v^{j}}{c}\right)^{2}}, \quad H^{j} \equiv \frac{d a^{j} / d t^{j}}{a^{j}} .
\end{gathered}
$$

The Einstein equations for the pressureless matter in the two regions are

$$
\left(H^{j}\right)^{2}+k^{j} c^{2} /\left(a^{j}\right)^{2}=\frac{8 \pi G}{3} \rho^{j}+\frac{1}{3} \Lambda c^{2},
$$

where $\rho^{j}$ is the mass density of matter $\left[\propto 1 /\left(a^{j}\right)^{3}\right]$.

The equations for the surface density $\sigma$ and the velocity $v^{\text {II }}$ of the shell are expressed as

$$
\begin{gathered}
\gamma^{\mathrm{II}} d\left(4 \pi R^{2} \sigma\right) / d t^{\mathrm{II}}=\left[4 \pi R^{2} \gamma^{2} v \rho\right]^{\mathrm{II}, \mathrm{I}}, \\
d\left(\gamma^{\mathrm{II}} v^{\mathrm{II}}\right) / d t^{\mathrm{II}}=-\gamma^{\mathrm{II}} v^{\mathrm{II}} H^{\mathrm{II}}+2 \pi G \sigma-\left[\gamma^{2} v^{2} \rho\right]^{\mathrm{II}} / \sigma,
\end{gathered}
$$

where $[\Phi]^{\mathrm{II}, \mathrm{I}} \equiv \Phi^{\mathrm{II}}-\Phi^{\mathrm{I}}$. The conditions of continuity of the metric $\left(d \tau^{2}=-d s^{2}\right)$ and the common velocity $d R / d \tau$ reduce to

$$
\begin{gathered}
d t^{\mathrm{I}} d d t^{\mathrm{II}}=\gamma^{\mathrm{I}} / \gamma^{\mathrm{II}}, \\
\gamma^{\mathrm{I}}\left(f^{\prime \mathrm{I}} v^{\mathrm{I}}+H^{\mathrm{I}} R\right)=\gamma^{\mathrm{II}}\left(f^{\prime \mathrm{II}} v^{\mathrm{II}}+H^{\mathrm{II}} R\right),
\end{gathered}
$$

where $f^{j}=d f^{j}\left(\chi^{j}\right) / d \chi^{j}$.

Another important component of jump conditions playing the role of a constraint equation is

$$
\left[\gamma\left(f^{\prime}+v H R\right)\right]^{\mathrm{II}, \mathrm{I}}=-4 \pi G \sigma R .
$$

Solving equations (4) and (5), we can obtain the time evolution of $\sigma$ and $v_{\mathrm{II}}$ in the shell, and $v^{\mathrm{I}}$ is derived using equation (7). These values of $\sigma, v^{\mathrm{I}}$, and $v^{\mathrm{II}}$ satisfy equation (8). The initial condition is given as a form of $\left(H^{\mathrm{I}}\right)_{i}=\left(H^{\mathrm{II}}\right)_{i}$ at an initial epoch $\left(t^{j}\right)_{i}$ such as the recombination epoch. 
The background models in $\mathrm{V}^{\mathrm{I}}$ and $\mathrm{V}^{\mathrm{II}}$ are rewritten using

$$
\begin{gathered}
y^{j} \equiv a^{j} /\left(a_{0}\right)^{j}, \quad \tau^{j} \equiv H_{0}^{j} t^{j}, \quad \lambda_{0}^{j} \equiv \frac{1}{3} \Lambda c^{2} /\left(H_{0}^{j}\right)^{2}, \\
\Omega_{0}^{j} \equiv \frac{8 \pi G}{3\left(H_{0}^{j}\right)^{2}}\left(\rho_{0}\right)^{j},
\end{gathered}
$$

where 0 denotes the present epoch (see Fig. 2). Equation (3) is given as

$$
d y^{j} / d \tau^{j}=\left(y^{j}\right)^{-1 / 2} P_{j}\left(y^{j}\right),
$$

where

$$
P_{j}\left(y^{j}\right) \equiv\left[\Omega_{0}^{j}+\lambda_{0}^{j}\left(y^{j}\right)^{3}+\left(1-\Omega_{0}^{j}-\lambda_{0}^{j}\right) y^{j}\right]^{1 / 2},
$$

and $\left(a_{0}\right)^{j}$ is given by

$$
\left(a_{0} H_{0}\right)^{j}=1 / \sqrt{1-\Omega_{0}^{j}-\lambda_{0}^{j}} .
$$

The conformal times $\eta^{j}$ are defined by

$$
\eta^{j} \equiv \sqrt{1-\Omega_{0}^{j}-\lambda_{0}^{j}} \int_{0}^{y^{j}} d y /\left[y^{1 / 2} P^{j}(y)\right] .
$$

The solution of equation (10) in the case of $k^{\mathrm{I}}=k^{\mathrm{II}}=-1$ and $\Lambda=0$ are expressed as

$$
\begin{gathered}
y^{j}=\frac{\Omega_{0}^{j}}{2\left(1-\Omega_{0}^{j}\right)}\left(\cosh \eta^{j}-1\right), \\
\tau^{j}=\frac{\Omega_{0}^{j}}{2\left(1-\Omega_{0}^{j}\right)^{3 / 2}}\left(\sinh \eta^{j}-\eta^{j}\right) .
\end{gathered}
$$

In the case of nonzero $\Lambda$, equation (10) is solved numerically.

Equations (4) and (5) were solved by Sakai et al. (1993), and it was shown in the case of $\Omega_{i} \simeq 1$ that the present value of $v^{\mathrm{II}}$ at $a / a_{i}>100$ is less than $100 \mathrm{~km} \mathrm{~s}^{-1}$, as long as the shell starts with the vanishing initial velocity $\left(v^{j}\right)_{i}=0$.

Here the initial condition $\left(H^{\mathrm{I}}\right)_{i}=\left(H^{\mathrm{II}}\right)_{i}$ at initial epoch $y^{j}=\left(y^{j}\right)_{i}$ is expressed as

$$
\begin{aligned}
& H_{0}^{\mathrm{I}}\left(y_{i}^{\mathrm{I}}\right)^{-3 / 2}\left[\Omega_{0}^{\mathrm{I}}+\lambda_{0}^{\mathrm{I}}\left(y_{i}^{\mathrm{I}}\right)^{3}+\left(1-\Omega_{0}^{\mathrm{I}}-\lambda_{0}^{\mathrm{I}}\right) y_{i}^{\mathrm{I}}\right]^{1 / 2} \\
= & H_{0}^{\mathrm{II}}\left(y_{i}^{\mathrm{II}}\right)^{-3 / 2}\left[\Omega_{0}^{\mathrm{II}}+\lambda_{0}^{\mathrm{II}}\left(y_{i}^{\mathrm{II}}\right)^{3}+\left(1-\Omega_{0}^{\mathrm{II}}-\lambda_{0}^{\mathrm{II}}\right) y_{i}^{\mathrm{II}}\right]^{1 / 2},
\end{aligned}
$$

where we have

$$
\frac{\Omega_{0}^{\mathrm{II}}}{\Omega_{0}^{\mathrm{I}}}=\frac{\rho_{0}^{\mathrm{II}}}{\rho_{0}^{\mathrm{I}}}\left(\frac{H_{0}^{\mathrm{I}}}{H_{0}^{\mathrm{II}}}\right)^{2}, \quad \frac{\lambda_{0}^{\mathrm{II}}}{\lambda_{0}^{\mathrm{I}}}=\left(\frac{H_{0}^{\mathrm{I}}}{H_{0}^{\mathrm{II}}}\right)^{2}
$$

from equation (9). If we eliminate $\Omega_{0}^{\mathrm{II}}, \lambda_{0}^{\mathrm{II}}$ from equation (16) using equation (17), we obtain

$$
\begin{aligned}
{\left[\frac{\rho_{0}^{\mathrm{II}}}{\rho_{0}^{\mathrm{I}}}\left(1-y_{i}^{\mathrm{II}}\right)\right.} & \left.-\left(\frac{y_{i}^{\mathrm{II}}}{y_{i}^{\mathrm{I}}}\right)^{3}\left(1-y_{i}^{\mathrm{I}}\right)\right] \Omega_{0}^{\mathrm{I}}-\left[1-\left(\frac{y_{i}^{\mathrm{II}}}{y_{i}}\right)^{2}\right] y_{i}^{\mathrm{II}} \lambda_{0}^{\mathrm{I}} \\
+ & {\left[\left(\frac{H_{0}^{\mathrm{II}}}{H_{0}^{\mathrm{I}}}\right)^{2}-\left(\frac{y_{i}^{\mathrm{II}}}{y_{i}^{\mathrm{I}}}\right)^{3}\right] y_{i}^{\mathrm{II}}=0 . }
\end{aligned}
$$

Since $y_{i}^{\mathrm{I}} \sim y_{i}^{\mathrm{II}} \sim 10^{-3}(\ll 1)$, we assume

$$
\frac{\rho_{0}^{\text {II }}}{\rho_{0}^{\mathrm{I}}}=\left(\frac{y_{i}^{\mathrm{II}}}{y_{i}^{\mathrm{I}}}\right)^{3}\left(1+\epsilon y_{i}^{\mathrm{I}}\right),
$$

where $\epsilon \approx 1$. Then we get from equation (18)

$$
\begin{aligned}
\left(\frac{H_{0}^{\mathrm{II}}}{H_{0}^{\mathrm{I}}}\right)^{2}= & \left(\frac{y_{i}^{\mathrm{II}}}{y_{i}^{\mathrm{I}}}\right)^{3}+\left[1-\left(\frac{y_{i}^{\mathrm{II}}}{y_{i}^{\mathrm{I}}}\right)^{2}\right] \lambda_{0}^{\mathrm{I}}+\left(\frac{y_{i}^{\mathrm{II}}}{y_{i}^{\mathrm{I}}}\right)^{3} \\
& \times\left[1-(1+\epsilon) \frac{y_{i}^{\mathrm{I}}}{y_{i}^{\mathrm{II}}} \Omega_{0}^{\mathrm{I}} .\right.
\end{aligned}
$$

If we give $\Omega_{0}^{\mathrm{I}}, \lambda_{0}^{\mathrm{I}}, \epsilon$, and $y_{i}^{\mathrm{II}} / y_{i}^{\mathrm{I}}$, we can obtain $H_{0}^{\mathrm{II}} / H_{0}^{\mathrm{I}}$ for equation (20) and derive $\rho_{0}^{\mathrm{II}} / \rho_{0}^{\mathrm{I}}$ from equation (20) and $\Omega_{0}^{\mathrm{II}} / \Omega_{0}^{\mathrm{I}}$ and $\lambda_{0}^{\mathrm{II}} / \lambda_{0}^{\mathrm{I}}$ from equation (17). From equations (17), (19), and (20), we find that $H_{0}^{\mathrm{II}} / H_{0}^{\mathrm{I}}<1$ and $\Omega_{0}^{\mathrm{II}} / \Omega_{0}^{\mathrm{I}}>1$ if

$$
\begin{aligned}
1> & \frac{y_{i}^{\mathrm{II}}}{y_{i}^{\mathrm{I}}}>1+\left[1-\left(\frac{y_{i}^{\mathrm{II}}}{y_{i}^{\mathrm{I}}}\right)^{2}\right]\left(\frac{y_{i}^{\mathrm{I}}}{y_{i}^{\mathrm{II}}}\right)^{3} \lambda_{0}^{\mathrm{I}} \\
& +\left[1-(1+\epsilon) \frac{y_{i}^{\mathrm{I}}}{y_{i}^{\mathrm{II}}}\right] \Omega_{0}^{\mathrm{I}} .
\end{aligned}
$$

In the case $\Lambda=0$, we have an example for $\Omega_{0}^{\mathrm{I}}=0.2$ :

$$
\begin{gathered}
\Omega_{0}^{\mathrm{II}}=0.56, \quad H_{0}^{\mathrm{II}} / H_{0}^{\mathrm{I}}=0.80, \quad \rho_{0}^{\mathrm{II}} / \rho_{0}^{\mathrm{I}}=1.8, \\
y_{i}^{\mathrm{II}} / y_{i}^{\mathrm{I}}=1.2, \quad \epsilon=4.1 .
\end{gathered}
$$

In the case $\Lambda \neq 0$, we have the example

$$
\begin{gathered}
\lambda_{0}^{\mathrm{I}}=0.672, \quad \lambda_{0}^{\mathrm{II}}=0.43, \quad \Omega_{0}^{\mathrm{I}}=0.3, \quad \Omega_{0}^{\mathrm{II}}=0.563, \\
H_{0}^{\mathrm{II}} / H_{0}^{\mathrm{I}}=0.80, \quad \epsilon=0.64,
\end{gathered}
$$

so that

$$
\Omega_{0}^{\mathrm{I}}+\lambda_{0}^{\mathrm{I}}=0.872, \quad \Omega_{0}^{\mathrm{II}}+\lambda_{0}^{\mathrm{II}}=0.993
$$

\subsection{Bulk Motion}

Now let us consider the velocity field around an observer $\mathrm{O}$ in $\mathrm{V}^{\mathrm{I}}$ at the point with $l_{0} \equiv(a \chi)_{0} \ll(a \chi)_{1}$. Since $l_{0}$ is much smaller than the curvature radius, we can approximately neglect the spatial curvature around him. Then he has the relative velocity

$$
\Delta v_{0}=\left(H_{0}^{\mathrm{I}}-H_{0}^{\mathrm{II}}\right) l_{0}
$$

to matter in the outer region in the direction of the $X$-axis. If $H_{0}^{\mathrm{I}}=100 h \mathrm{~km} \mathrm{~s}^{-1} \mathrm{Mpc}^{-1}, H_{0}^{\mathrm{II}}=0.82 H_{0}^{\mathrm{I}}$, and $l_{0}=40$ $h^{-1} \mathrm{Mpc}$, we have $\Delta v_{0}=720 \mathrm{~km} \mathrm{~s}^{-1}$.

Here consider a galaxy $\mathrm{G}$ with the radius coordinate $\chi$ and angle $\varphi$. Then the relative velocity of $\mathrm{G}$ to matter in the outer region is

$$
\Delta v_{G}=\left(H_{0}^{\mathrm{I}}-H_{0}^{\mathrm{II}}\right)\left(a_{0} \chi\right)
$$

in the radial direction from the center $\mathrm{C}$ of the inner region (Fig. 3).

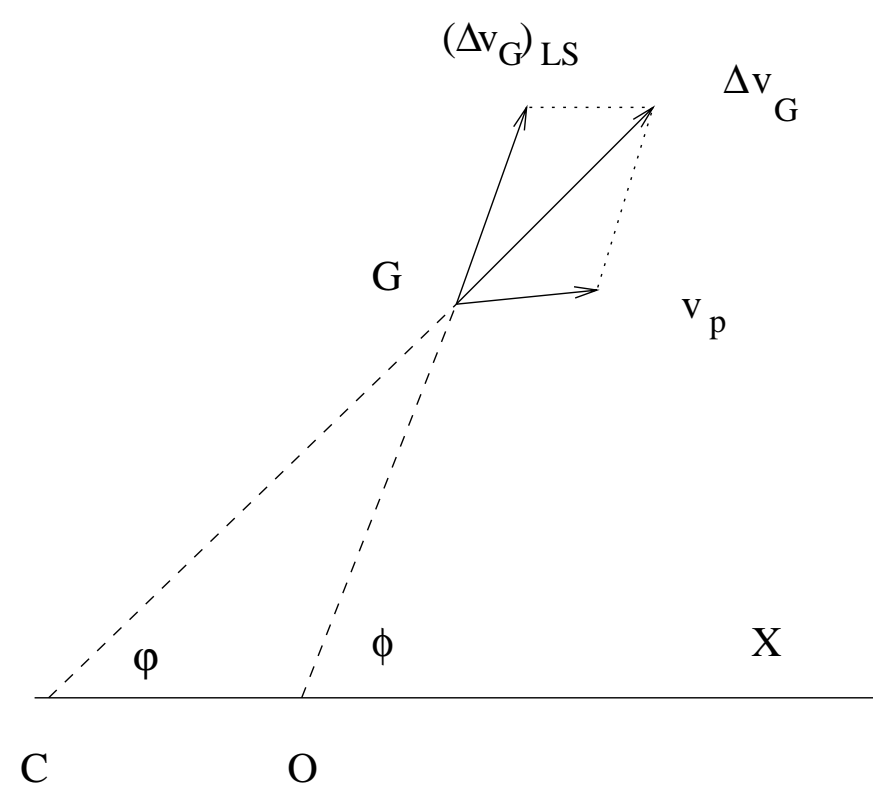

FIG. 3.-Components of the bulk velocity 
This velocity can be divided into the $X$ component $\left(\Delta v_{G}\right)_{X}$ and the line-of-sight component $\left(\Delta v_{G}\right)_{L S}$ with respect to the observer $\mathrm{O}$ as follows, noticing that the angle $\angle \mathrm{GOX}(=\phi)$ satisfies the relation

$$
\begin{gathered}
\sin \phi=\chi \sin \varphi /\left(\chi^{2}+\chi_{0}^{2}-2 \chi \chi_{0} \cos \varphi\right)^{1 / 2}, \\
\cot \phi=\left(\cos \varphi-\chi_{0} / \chi\right) / \sin \varphi .
\end{gathered}
$$

Their values are

$$
\begin{aligned}
\left(\Delta v_{G}\right)_{X} & =\Delta v_{G} \sin (\phi-\varphi) / \sin \phi=\Delta v_{0}, \\
\left(\Delta v_{G}\right)_{L S} & =\Delta v_{G} \sin \varphi / \sin \phi \\
& =\Delta v_{G}\left[1-2\left(\chi_{0} / \chi\right) \cos \varphi+\left(\chi_{0} / \chi\right)^{2}\right]^{1 / 2} \\
& \simeq \Delta v_{G} .
\end{aligned}
$$

That is, the $X$ component $\left(\Delta v_{G}\right)_{X}$ is constant and equal to $\Delta v_{0}$, and another component, which is in the line-of-sight direction from the observer, is nearly equal to $\Delta v_{G}$. Because the first component $\left(\Delta v_{G}\right)_{X}$ is independent of the position, it can be interpreted as the peculiar velocity $v_{p}$ of galaxies which represents the bulk motion:

$$
v_{p}=\left(\Delta v_{G}\right)_{X}=\Delta v_{0} .
$$

\section{REDSHIFT FORMULA AND THE CMB ANISOTROPY}

The wavevector $k^{\mu}$ in the inner and outer regions in the plane of $\theta=\pi / 2$ is obtained by solving a null geodesic equation and is expressed as

$$
\begin{gathered}
\left(k^{0}\right)^{j}=d t^{j} / d \lambda=\frac{a_{0}^{j}}{a^{j}} w_{1}^{j}, \\
\left(k^{\chi}\right)^{j}=d \chi^{j} / d \lambda= \pm \frac{a_{0}^{j} w_{1}^{j}}{\left(a^{j}\right)^{2}}\left[1-\left(\frac{d^{j} / w_{1}^{j}}{a_{0}^{j} \sinh \chi^{j}}\right)^{2}\right]^{1 / 2}, \\
\left(k^{\varphi}\right)^{j}=d \varphi / d \lambda=d^{j} /\left(a^{j} \sinh \chi^{j}\right)^{2},
\end{gathered}
$$

where $j=\mathrm{I}$ and II, and $\lambda$ is an affine parameter.

\subsection{Light Paths}

In the inner region $\mathrm{V}^{\mathrm{I}}$, it is assumed that at the present epoch $\left(t^{\mathrm{I}}=t_{0}^{\mathrm{I}}\right)$ all rays reach an observer at the point $\mathrm{O}$ with $\chi=\chi_{0}, \theta=\pi / 2$, and $\varphi=0$ in the $X$-axis, and the angle between the rays and the $X$-axis is $\phi$. Then we have

$$
\phi=\phi_{1}, \quad \pi-\phi_{1},
$$

where

$$
\phi_{1} \equiv \sin ^{-1}\left(\frac{d^{\mathrm{I}} / w_{1}^{\mathrm{I}}}{a_{0}^{\mathrm{I}} \sinh \chi_{0}^{\mathrm{I}}}\right) \quad(<\pi / 2) .
$$

For $\phi=\phi_{1}$, the rays are expressed as

$$
\begin{aligned}
G\left(\chi^{\mathrm{I}}\right) & \equiv \cosh ^{-1}\left(\frac{\cosh \chi^{\mathrm{I}}}{h_{0}^{\mathrm{I}}}\right)-\cosh ^{-1}\left(\frac{\cosh \chi_{0}^{\mathrm{I}}}{h_{0}^{\mathrm{I}}}\right) \\
& =\eta_{0}^{\mathrm{I}}-\eta^{\mathrm{I}},
\end{aligned}
$$

where

$$
h_{0}^{j} \equiv\left\{1+\left[\frac{d^{j} / w_{1}^{j}}{\left(a_{0}\right)^{j}}\right]^{2}\right\}^{1 / 2},
$$

$\eta^{\mathrm{I}}$ is defined by equations (13) and (14), and $\eta_{0}^{\mathrm{I}}$ is equal to $\eta^{\mathrm{I}}$ at present epoch $\left(y^{\mathrm{I}}=1\right)$.
For $\phi=\pi-\phi_{1}$, we have

$$
\begin{aligned}
G\left(\chi^{\mathrm{I}}\right) & =-\eta_{0}^{\mathrm{I}}+\eta^{\mathrm{I}}, \quad \text { for } \eta_{0}^{\mathrm{I}} \geq \eta^{\mathrm{I}}>\eta_{m}, \\
& =-\eta^{\mathrm{I}}-\eta_{0}^{\mathrm{I}}+2 \eta_{m}, \quad \text { for } \eta^{\mathrm{I}} \leq \eta_{m},
\end{aligned}
$$

where

$$
\eta_{m} \equiv \eta_{0}^{\mathrm{I}}-\cosh ^{-1}\left(\frac{\cosh \chi_{0}^{\mathrm{I}}}{h_{0}^{\mathrm{I}}}\right) .
$$

In the latter case, $\chi^{\mathrm{I}}$ has the minimum value (i.e., $k^{\chi}=0$ ) at $\eta^{\mathrm{I}}=\eta_{m}$.

At the boundary, $\eta^{\mathrm{I}}=\eta_{1}^{\mathrm{I}}$ and $\chi^{\mathrm{I}}=\chi_{1}^{\mathrm{I}}$, therefore, we obtain

$$
\eta_{1}^{\mathrm{I}}=\eta_{0}^{\mathrm{I}} \pm \cosh ^{-1}\left(\frac{\cosh \chi_{0}^{\mathrm{I}}}{h_{0}^{\mathrm{I}}}\right)-\cosh ^{-1}\left(\frac{\cosh \chi_{1}^{\mathrm{I}}}{h_{0}^{\mathrm{I}}}\right)
$$

for

$$
\phi=\left(\begin{array}{c}
\phi_{1} \\
\pi-\phi_{1}
\end{array}\right),
$$

respectively. In the outer region $\mathrm{V}^{\mathrm{II}}$, we have

$$
\begin{aligned}
G\left(\chi^{\mathrm{II}}\right) & \equiv \cosh ^{-1}\left(\frac{\cosh \chi^{\mathrm{II}}}{h_{0}^{\mathrm{II}}}\right)-\cosh ^{-1}\left(\frac{\cosh \chi_{1}^{\mathrm{II}}}{h_{0}^{\mathrm{II}}}\right) \\
& =\eta_{1}^{\mathrm{II}}-\eta^{\mathrm{II}},
\end{aligned}
$$

where $\eta^{\mathrm{II}}$ is given by equations (13) and (14), and $\eta_{1}^{\mathrm{II}}$ and $\chi_{1}^{\mathrm{II}}$ are the values at the shell. At the recombination epoch we have

$$
\begin{aligned}
\eta_{\mathrm{rec}}^{\mathrm{II}}= & \eta_{1}^{\mathrm{II}}-\cosh ^{-1}\left(\frac{\cosh \chi_{\mathrm{rec}}^{\mathrm{II}}}{h_{0}^{\mathrm{II}}}\right) \\
& +\cosh ^{-1}\left(\frac{\cosh \chi_{1}^{\mathrm{II}}}{h_{0}^{\mathrm{II}}}\right) .
\end{aligned}
$$

The junction of wavevectors at the boundary is expressed as

$$
\begin{aligned}
\left(k^{0}\right)^{\mathrm{I}} & =\left(k^{0}\right)^{\mathrm{II}}, \\
\left(\sqrt{g_{\chi \chi}} k^{\chi}\right)^{\mathrm{I}} & =\left(\sqrt{g_{\chi \chi}} k^{\chi}\right)^{\mathrm{II}}, \\
\left(\sqrt{g_{\varphi \varphi}} k^{\varphi}\right)^{\mathrm{I}} & =\left(\sqrt{g_{\varphi \varphi}} k^{\varphi}\right)^{\mathrm{II}} .
\end{aligned}
$$

Equations (43) and (45) give

$$
\begin{aligned}
\left(\frac{a_{0}}{a_{1}}\right)^{\mathrm{I}} w_{1}^{\mathrm{I}} & =\left(\frac{a_{0}}{a_{1}}\right)^{\mathrm{II}} w_{1}^{\mathrm{II}}, \\
d^{\mathrm{I}} & =d^{\mathrm{II}},
\end{aligned}
$$

respectively, where we used the relation $R=(a f)^{\mathrm{I}}=(a f)^{\mathrm{II}}$. The conditions given by equations (43) and (45) are evidently consistent with equation (44), since $k^{\mu}$ is a null vector.

\subsection{Redshift Formula}

Now let us derive the redshift formula for rays which are emitted at the recombination epoch. Here this epoch is defined as the time of the radiation temperature $T_{r}=$ $10^{3}\left(T_{r}\right)_{0}$ in the region $\mathrm{V}^{\mathrm{II}}$, where $\left(T_{r}\right)_{0}$ is the present temperature $(\simeq 2.7 \mathrm{~K})$. The total redshift factor $\left(1+z_{\mathrm{rec}}\right)$ is calculated as the product of two redshift factors which are caused in the two regions $\mathrm{V}^{\mathrm{I}}$ and $\mathrm{V}^{\mathrm{II}}$.

First, we assume that the shell is comoving, and later the correction due to the motion of the shell is examined. If we consider a virtual observer at the center $\mathrm{C}(\chi=0)$, a light ray which is received by him at $\bar{\eta}^{\mathrm{I}}=\bar{\eta}_{0}^{\mathrm{I}}$ is expressed as

$$
\bar{\eta}_{0}^{\mathrm{I}}-\bar{\eta}^{\mathrm{I}}=\chi^{\mathrm{I}}, \quad \bar{\eta}_{0}^{\mathrm{I}}-\bar{\eta}_{1}^{\mathrm{I}}=\chi_{1}^{\mathrm{I}}
$$


TABLE 1

Dipole and Quadrupole Moments and the Velocity $v_{d}$ IN Single-Shell Models

\begin{tabular}{ccccccccr}
\hline \hline$\Omega_{0}^{\mathrm{I}}$ & $\Omega_{0}^{\text {II }}$ & $\lambda_{0}^{\mathrm{I}}$ & $\lambda_{0}^{\text {II }}$ & $h^{\mathrm{I}}$ & $h^{\mathrm{II}} / h^{\mathrm{I}}$ & $\begin{array}{c}D \\
\left(\times 10^{4}\right)\end{array}$ & $\begin{array}{c}Q \\
\left(\times 10^{5}\right)\end{array}$ & $\begin{array}{c}v_{d} \\
\left(\mathrm{~km} \mathrm{~s}^{-1}\right)\end{array}$ \\
\hline $0.2 \ldots \ldots$ & 0.56 & 0.0 & 0.0 & 0.7 & 0.82 & 5.56 & -2.87 & $81.8^{\mathrm{a}}$ \\
$0.2 \ldots \ldots$ & 0.88 & 0.0 & 0.0 & 0.7 & 0.82 & 8.27 & -4.27 & $121.5^{\mathrm{a}}$ \\
$0.2 \ldots \ldots$ & 0.56 & 0.0 & 0.0 & 0.7 & 0.90 & 5.38 & -2.79 & $79.1^{\mathrm{a}}$ \\
$0.2 \ldots \ldots$ & 0.88 & 0.0 & 0.0 & 0.7 & 0.90 & 8.21 & -4.23 & $120.7^{\mathrm{a}}$ \\
$0.3 \ldots \ldots$ & 0.56 & 0.0 & 0.0 & 0.7 & 0.82 & 3.90 & -2.04 & $57.3^{\mathrm{a}}$ \\
$0.3 \ldots \ldots$ & 0.88 & 0.0 & 0.0 & 0.7 & 0.82 & 6.66 & -3.39 & $97.9^{\mathrm{a}}$ \\
$0.2 \ldots \ldots$ & 0.56 & 0.672 & 0.43 & 0.7 & 0.82 & 7.92 & -3.74 & $111.4^{\mathrm{a}}$ \\
$0.2 \ldots \ldots$ & 0.56 & 0.0 & 0.0 & 0.7 & 0.82 & 5.55 & -1.91 & $81.6^{\mathrm{b}}$ \\
$0.2 \ldots \ldots$ & 0.88 & 0.0 & 0.0 & 0.7 & 0.82 & 8.21 & -2.68 & $120.7^{\mathrm{b}}$ \\
$0.2 \ldots \ldots$ & 0.56 & 0.0 & 0.0 & 0.7 & 0.82 & 8.07 & -6.35 & $118.6^{\mathrm{c}}$ \\
$0.2 \ldots \ldots$ & 0.88 & 0.0 & 0.0 & 0.7 & 0.82 & 12.31 & -9.62 & $181.0^{\mathrm{c}}$ \\
$0.2 \ldots \ldots$ & 0.56 & 0.0 & 0.0 & 0.7 & 0.82 & 5.57 & -2.87 & $81.8^{\mathrm{d}}$ \\
$0.2 \ldots \ldots$ & 0.88 & 0.0 & 0.0 & 0.7 & 0.82 & 8.27 & -4.27 & $121.6^{\mathrm{d}}$ \\
\hline
\end{tabular}

a $\bar{z}_{1}^{\mathrm{I}}=0.067, l_{0}=40\left(h^{\mathrm{I}}\right)^{-1}$.

b $\bar{z}_{1}^{\mathrm{I}}=0.1, l_{0}=40\left(h^{\mathrm{I}}\right)^{-1}$.

c $\bar{z}_{1}^{\mathrm{I}}=0.067, l_{0}=60\left(h^{\mathrm{I}}\right)^{-1}$

d $\bar{z}_{1}^{\mathrm{I}}=0.067, l_{0}=40\left(h^{\mathrm{I}}\right)^{-1}$, and the shell velocity is $200 \mathrm{~km} \mathrm{~s}^{-1}$.

in $\mathrm{V}^{\mathrm{I}}$ and

$$
\bar{\eta}_{\mathrm{rec}}^{\mathrm{II}}-\bar{\eta}^{\mathrm{II}}=\chi_{\mathrm{rec}}^{\mathrm{II}}-\chi^{\mathrm{II}}, \quad \bar{\eta}_{\mathrm{rec}}^{\mathrm{II}}-\bar{\eta}_{1}^{\mathrm{II}}=\chi_{\mathrm{rec}}^{\mathrm{II}}-\chi_{1}^{\mathrm{II}}
$$

in $\mathrm{V}^{\mathrm{II}}$, when the ray is emitted at the recombination epoch.

The redshift factors are

$$
1+\bar{z}_{1}^{\mathrm{I}}=\frac{a_{0}^{\mathrm{I}}}{a^{\mathrm{I}}\left(\bar{\eta}_{1}^{\mathrm{I}}\right)}=\frac{1}{y^{\mathrm{I}}\left(\bar{\eta}_{1}^{\mathrm{I}}\right)}
$$

in $\mathrm{V}^{\mathrm{I}}$, and

$$
\frac{1+\bar{z}_{\mathrm{rec}}^{\mathrm{II}}}{1+\bar{z}_{1}^{\mathrm{II}}}=\frac{a^{\mathrm{II}}\left(\bar{\eta}_{1}^{\mathrm{II}}\right)}{a^{\mathrm{II}}\left(\bar{\eta}_{\mathrm{rec}}^{\mathrm{II}}\right)}=\frac{y^{\mathrm{II}}\left(\bar{\eta}_{1}^{\mathrm{II}}\right)}{y^{\mathrm{II}}\left(\bar{\eta}_{\mathrm{rec}}^{\mathrm{II}}\right)}
$$

in $\mathrm{V}^{\mathrm{II}}$. The junction condition in equation (6) gives $\bar{z}_{1}^{\mathrm{I}}=\bar{z}_{1}^{\mathrm{II}}$ for $v^{\mathrm{I}}=v^{\mathrm{II}}=0$, so that

$$
1+\bar{z}_{\mathrm{rec}}^{\mathrm{II}}=\frac{y^{\mathrm{II}}\left(\bar{\eta}_{1}^{\mathrm{II}}\right)}{y^{\mathrm{II}}\left(\bar{\eta}_{\mathrm{rec}}^{\mathrm{II}}\right) y^{\mathrm{I}}\left(\bar{\eta}_{1}^{\mathrm{I}}\right)} .
$$

Here we specify the value of $\bar{z}_{1}^{\mathrm{I}}$ as $\bar{z}_{1}^{\mathrm{I}}=0.067 \sim 0.1$. Then $\bar{\eta}_{1}^{\mathrm{I}}$ and $\chi_{1}^{\mathrm{I}}$ are determined from equations (48) and (50), and $\bar{\eta}_{1}^{\mathrm{II}}$ and $\chi_{1}^{\mathrm{II}}$ are determined using the relations

$$
a_{0}^{\mathrm{I}} y^{\mathrm{I}}\left(\bar{\eta}_{1}^{\mathrm{I}}\right) \sinh \chi_{1}^{\mathrm{I}}=a_{0}^{\mathrm{II}} y^{\mathrm{II}}\left(\bar{\eta}_{1}^{\mathrm{II}}\right) \sinh \chi_{1}^{\mathrm{II}}
$$

and

$$
a_{0}^{\mathrm{I}} \int_{0}^{\bar{\eta}_{1} \mathrm{I}} y^{\mathrm{I}}\left(\eta^{\mathrm{I}}\right) d \eta^{\mathrm{I}}=a_{0}^{\mathrm{II}} \int_{0}^{\bar{\eta}_{1} \mathrm{II}} y^{\mathrm{II}}\left(\eta^{\mathrm{II}}\right) d \eta^{\mathrm{II}},
$$

which are obtained from equations (2) and (6). If $\bar{z}_{\text {rec }}^{\mathrm{II}}$ is moreover specified (in the following we take the value $\left.\bar{z}_{\text {rec }}^{\text {II }}=10^{3}-1\right), y\left(\bar{\eta}_{\text {rec }}\right)$ or $\bar{\eta}_{\text {rec }}$ is determined using equation (52).

Next we consider the observer at $\mathrm{O}$ (with $\chi^{\mathrm{I}}=\chi_{0}^{\mathrm{I}}$ and $\varphi=0)$. The above-determined $\chi_{1}^{j}(j=\mathrm{I}$ and II $)$ and $\eta_{\mathrm{rec}}^{\mathrm{II}}$ $\left(=\bar{\eta}_{\mathrm{rec}}^{\mathrm{II}}\right)$ are used also for rays reaching this observer, to identify the position of the shell and the recombination

TABLE 2

Dipole and Quadrupole Moments AND the Velocity $v_{d}$ IN Double-Shell MODELS IN THE CASE OF $\lambda_{0}^{j}=0$ AND $l_{0}=40\left(h^{\mathrm{I}}\right)^{-1}$

\begin{tabular}{ccccccccc}
\hline \hline$\Omega_{0}^{\mathrm{I}}$ & $\Omega_{0}^{\mathrm{II}}$ & $\Omega_{0}^{\mathrm{III}}$ & $h^{\mathrm{I}}$ & $h^{\mathrm{II}} / h^{\mathrm{I}}$ & $h^{\mathrm{III}} / h^{\mathrm{I}}$ & $\begin{array}{c}D \\
\left(\times 10^{4}\right)\end{array}$ & $\begin{array}{c}Q \\
\left(\times 10^{5}\right)\end{array}$ & $\begin{array}{c}v_{d} \\
\left(\mathrm{~km} \mathrm{~s}^{-1}\right)\end{array}$ \\
\hline $0.2 \ldots \ldots$ & 0.36 & 0.56 & 0.7 & 0.92 & 0.82 & 5.55 & -2.37 & $81.6^{\mathrm{a}}$ \\
$0.2 \ldots \ldots$ & 0.36 & 0.56 & 0.7 & 0.92 & 0.82 & 5.52 & -1.44 & $81.1^{\mathrm{b}}$ \\
\hline
\end{tabular}

a $\bar{z}_{1}^{\mathrm{I}}=0.067, \bar{z}_{1}^{\mathrm{II}}=0.1$.

b $\bar{z}_{1}^{\mathrm{I}}=0.1, \bar{z}_{1}^{\mathrm{II}}=0.2$.

TABLE 3

Dipole and Quadrupole Moments AND the Velocity $v_{d}$ IN THE Model With A Self-Similar Region IN THE CASE OF $\lambda_{0}^{j}=0$ AND $l_{0}=40\left(h^{\mathrm{I}}\right)^{-1}$

\begin{tabular}{ccccccc}
\hline \hline$\Omega_{0}^{\mathrm{I}}$ & $\Omega_{0}^{\mathrm{III}}$ & $h^{\mathrm{I}}$ & $h^{\mathrm{III}} / h^{\mathrm{I}}$ & $\begin{array}{c}D \\
\left(\times 10^{4}\right)\end{array}$ & $\begin{array}{c}Q \\
\left(\times 10^{5}\right)\end{array}$ & $\begin{array}{c}v_{d} \\
\left(\mathrm{~km} \mathrm{~s}^{-1}\right)\end{array}$ \\
\hline $0.2 \ldots \ldots \ldots \ldots \ldots$ & 0.89 & 0.7 & 0.81 & 8.40 & -4.41 & $123.4^{\mathrm{a}}$ \\
$0.2 \ldots \ldots \ldots \ldots \ldots$ & 0.72 & 0.7 & 0.83 & 7.40 & -2.61 & $108.8^{\mathrm{b}}$ \\
$0.3 \ldots \ldots \ldots \ldots \ldots$ & 0.91 & 0.7 & 0.84 & 8.08 & -4.11 & $118.8^{\mathrm{a}}$ \\
$0.3 \ldots \ldots \ldots \ldots \ldots$ & 0.80 & 0.7 & 0.86 & 6.94 & -2.25 & $102.0^{\mathrm{b}}$ \\
\hline
\end{tabular}

a $\bar{z}_{1}^{\mathrm{I}}=0.067, \bar{z}_{1}^{\mathrm{II}}=0.1$.

b $\bar{z}_{1}^{\mathrm{I}}=0.1, \bar{z}_{1}^{\mathrm{II}}=0.2$. 
epoch. It should be noted that $\eta_{\mathrm{rec}}^{\mathrm{II}}$ depends only on the temperature at the recombination epoch and is independent of the existence of the inner region $\mathrm{V}^{\mathrm{I}}$.

In $\mathrm{V}^{\mathrm{I}}$ we have the redshift

$$
1+z_{1}^{\mathrm{I}}=\frac{a_{0}^{\mathrm{I}}}{a^{\mathrm{I}}\left(\eta_{1}^{\mathrm{I}}\right)}=\frac{1}{y^{\mathrm{I}}\left(\eta_{1}^{\mathrm{I}}\right)},
$$

and in $\mathrm{V}^{\mathrm{II}}$ we have

$$
\frac{1+z_{\mathrm{rec}}^{\mathrm{II}}}{1+z_{1}^{\mathrm{II}}}=\frac{a^{\mathrm{II}}\left(\eta_{1}^{\mathrm{II}}\right)}{a^{\mathrm{II}}\left(\eta_{\mathrm{rec}}^{\mathrm{II}}\right)}=\frac{y^{\mathrm{II}}\left(\eta_{1}^{\mathrm{II}}\right)}{y^{\mathrm{II}}\left(\eta_{\mathrm{rec}}^{\mathrm{II}}\right)} .
$$

For a given $\chi_{1}^{\mathrm{I}}$, we obtain $\eta_{1}^{\mathrm{I}}$ by solving equation (40) and obtain $z_{1}^{\mathrm{I}}$ from equation (55). The junction condition in equation (6) gives $z_{1}^{\mathrm{I}}=z_{1}^{\mathrm{II}}$, and $\eta_{1}^{\mathrm{II}}$ is related to $\eta_{1}^{\mathrm{I}}$ using the relations given in equations (53) and (54). Then the product of equations (55) and (56) reduces to

$$
1+z_{\mathrm{rec}}^{\mathrm{II}}=\frac{y^{\mathrm{II}}\left(\eta_{1}^{\mathrm{II}}\right)}{y^{\mathrm{I}}\left(\eta_{1}^{\mathrm{I}}\right) y^{\mathrm{II}}\left(\eta_{\mathrm{rec}}^{\mathrm{II}}\right)},
$$

which is given as a function of angle $\phi$.

Now let us consider the case when the shell is not comoving, i.e., $v^{\mathrm{I}} \neq 0, v^{\mathrm{II}} \neq 0$. As was shown by Sakai et al. (1993), the velocities are greater than $200 \mathrm{~km} \mathrm{~s}^{-1}$, and $\left(v^{j} / c\right)^{2}<10^{-7}$ for $j=\mathrm{I}$ and II. Accordingly, we can assume $\gamma^{j}=1$ for $j=\mathrm{I}$ and II, so that the condition $z_{1}^{\mathrm{I}}=z_{1}^{\mathrm{II}}$ and equation (54) hold. In order to take the shell motion into account, we assume the relation

$$
\chi_{1}^{\mathrm{I}}=\left(\chi_{1}^{\mathrm{I}}\right)_{\phi=0}+v^{\mathrm{I}}\left[\eta_{1}^{\mathrm{I}}-\left(\eta_{1}^{\mathrm{I}}\right)_{\phi=0}\right]
$$

in equation (40) for arbitrary $\phi$, where $\left(\chi_{1}^{\mathrm{I}}\right)_{\phi=0}$ and $\left(\eta_{1}^{\mathrm{I}}\right)_{\phi=0}$ are their values for the ray incident in the direction of $\phi=0$. Subsequent calculations are the same as the calculations for $v^{\mathrm{I}}=0$.

The derivations of $z_{\mathrm{rec}}^{\mathrm{II}}$ in the case of double shells and in the inhomogeneous model with an intermediate self-similar region are shown in Appendixes A and B.

\section{3. $C M B$ Anisotropy}

The values of $z_{\text {rec }}^{\mathrm{II}}$ are numerically calculated for $0<\phi<\pi$, and their dipole and quadrupole moments are derived. When $z_{\mathrm{rec}}\left[=z_{\mathrm{rec}}^{\mathrm{II}}(\phi)\right]$ is given, the temperature $T(\phi)$ of the cosmic background radiation is proportional to $1 /\left(1+z_{\text {rec }}\right)$, and the dipole moment $D$ and quadrupole moment $Q$ are defined as

$$
\begin{aligned}
& D \equiv\left|\int_{0}^{\pi} \int_{0}^{2 \pi}\left(1+z_{\mathrm{rec}}\right)^{-1} Y_{10} \sin \phi d \phi d \varphi\right|\left\langle\left(1+z_{\mathrm{rec}}\right)^{-1}\right\rangle, \\
& Q \equiv\left|\int_{0}^{\pi} \int_{0}^{2 \pi}\left(1+z_{\mathrm{rec}}\right)^{-1} Y_{20} \sin \phi d \phi d \varphi\right|\left\langle\left(1+z_{\mathrm{rec}}\right)^{-1}\right\rangle,
\end{aligned}
$$

where $\langle>$ means the average value taken over the whole sky and

$$
\begin{aligned}
Y_{10}(\phi) & =\sqrt{\frac{3}{4 \pi}} \cos \phi, \\
Y_{20}(\phi) & =\sqrt{\frac{5}{4 \pi}}\left(\frac{3}{2} \cos ^{2} \phi-\frac{1}{2}\right) .
\end{aligned}
$$

The Doppler velocity $v_{d}$ corresponding to $D$ is given by

$$
v_{d} \equiv c\left[(3 / 4 \pi)^{1 / 2} D\right] \text {. }
$$

Assuming $l_{0}\left(\equiv a_{0} \chi_{0}\right)=40\left(h^{\mathrm{I}}\right)^{-1} \mathrm{Mpc}$ mainly, $D, Q$, and $v_{d}$ were derived for various model parameters. Their values in models with a single shell and double shells are shown in Tables 1 and 2, respectively. The values in models with an intermediate self-similar region are shown in Table 3. In Table 1 the values in the case with a moving shell are shown.

It is found from Table 1 that (1) $v_{d} / v_{p}$ is smaller than 0.17 in all cases, (2) $v_{d}$ is approximately proportional to $l_{0}$ (as well as $\left.v_{p}\right)$, (3) $v_{d}$ is smaller for smaller $\left(\Omega_{0}^{\mathrm{II}}-\Omega_{0}^{\mathrm{I}}\right)$ and $\left(h^{\mathrm{I}}-h^{\mathrm{II}}\right)$, (4) the positive cosmological constant plays a role of increasing $v_{d}$, and (5) the influence of the shell motion on $D, Q$, and $v_{d}$ is negligibly small.

If we compare two lines in Table 2 with the corresponding ones (the first line and eighth line) in Table 1, the results in the models with a single shell and double shells are found to be quite consistent. Moreover, if we compare four lines in Table 3 with the corresponding ones (the second, fourth, and sixth lines) in Table 1, the results in the models with a single shell and a self-similar region are found to be similarly consistent. Accordingly, $v_{d} / v_{p}$ is $\approx 0.1$ in all models we treated here.

\section{NAIVE DERIVATION OF REDSHIFT FACTORS}

For the dipole anisotropy the maximum difference of temperatures and redshifts can be seen in a direction $(\phi=$ $\left.\phi_{1}\right)$ and the inverse direction $\left(\phi=\pi-\phi_{1}\right)$. Here let us compare the redshifts in the directions $\phi=0$ and $\pi$ appearing in the model with a single shell. The spatial curvature is neglected for simplicity. In the $X$-axis we consider six points $\mathrm{O}, \mathrm{A}, \mathrm{B}, \mathrm{C}, \mathrm{D}$, and $\mathrm{E}$, for which $X=\chi_{0}, \chi_{1}, \chi_{1}+2 \chi_{0}, 0$, $-\chi_{1}$, and $-\chi_{1}+2 \chi_{0}$, as shown in Figure 4 .

Points B and D have the equal distance from the observer's point $O$.

The redshifts $z_{\text {rec }}(0)$ and $z_{\text {rec }}(\pi)$ of the rays from other points $\mathbf{P}$ and $\mathbf{P}^{\prime}$ at the recombination epoch to the observer at $\mathrm{O}$ in the directions $\phi=0$ and $\pi$ are divided into three steps: $(\mathrm{P} \rightarrow \mathrm{B}, \mathrm{B} \rightarrow \mathrm{A}, \mathrm{A} \rightarrow \mathrm{O})$ and $\left(\mathrm{P}^{\prime} \rightarrow \mathrm{D}, \mathrm{D} \rightarrow \mathrm{E}, \mathrm{E} \rightarrow\right.$ $\mathrm{O})$,respectively. That is,

$$
\begin{aligned}
& 1+z_{\mathrm{rec}}(0)=\left(1+z_{\mathrm{PB}}\right)\left(1+z_{\mathrm{BA}}\right)\left(1+z_{\mathrm{AO}}\right), \\
& 1+z_{\mathrm{rec}}(\pi)=\left(1+z_{\mathrm{P}^{\prime} \mathrm{D}}\right)\left(1+z_{\mathrm{DE}}\right)\left(1+z_{\mathrm{EO}}\right) .
\end{aligned}
$$

Among these three steps, the first and third have equal redshifts evidently: $z_{\mathrm{PB}}=z_{\mathrm{P}^{\prime} \mathrm{D}}, z_{\mathrm{AO}}=z_{\mathrm{EO}}$. In the processes $(\mathrm{B} \rightarrow \mathrm{A})$ and $(\mathrm{D} \rightarrow \mathrm{E})$, we have the redshifts due to the

$$
\text { V II }
$$

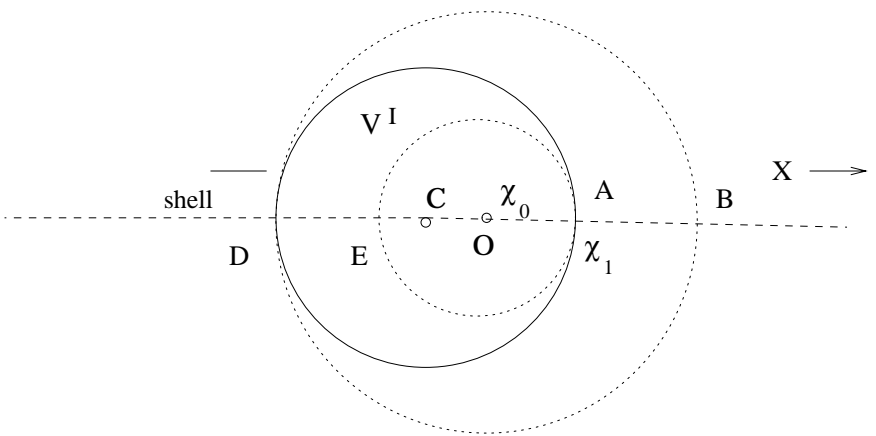

FIG. 4.-Diagram for the naive derivation 
cosmic expansion, the Doppler shift, and the gravitational redshift. The expansion redshift factors in $\mathrm{V}^{\mathrm{II}}$ and $\mathrm{V}^{\mathrm{I}}$ are

$$
\left[1+H_{0}^{\mathrm{II}}\left(2 a_{0} \chi_{0} / c\right)\right], \quad\left[1+H_{0}^{\mathrm{I}}\left(2 a_{0} \chi_{0} / c\right)\right],
$$

respectively. The Doppler shifts at A and D due to the relative velocity between $\mathrm{V}^{\mathrm{I}}$ and $\mathrm{V}^{\mathrm{II}}$ frames are

$$
\begin{aligned}
& {\left[1-\frac{1}{c}\left(H_{0}^{\mathrm{I}}-H_{0}^{\mathrm{II}}\right) a_{0}\left(\chi_{1}-\chi_{0}\right)\right],} \\
& {\left[1-\frac{1}{c}\left(H_{0}^{\mathrm{I}}-H_{0}^{\mathrm{II}}\right) a_{0}\left(\chi_{1}+\chi_{0}\right)\right],}
\end{aligned}
$$

respectively. The gravitational redshifts are represented by potentials $\psi_{\mathrm{BA}}$ and $\psi_{\mathrm{DE}}$, given by

$$
\begin{aligned}
& \psi_{\mathrm{BA}} \equiv \frac{4 \pi G\left(a_{0}\right)^{3}}{c^{2}}\left[\frac{1}{3} \frac{\rho_{0}^{\mathrm{I}}\left(\chi_{1}\right)^{3}}{\chi_{1}+\chi_{0}}+\frac{\rho_{0}^{\mathrm{II}}\left(\chi_{1}+\chi_{0}\right)^{2} \chi_{0}}{\chi_{1}+\chi_{0}}\right], \\
& \psi_{\mathrm{DE}} \equiv \frac{4 \pi G\left(a_{0}\right)^{3}}{c^{2}}\left[\frac{1}{3} \frac{\rho_{0}^{\mathrm{I}}\left(\chi_{1}\right)^{3}}{\chi_{1}+\chi_{0}}-\frac{\rho_{0}^{\mathrm{II}}\left(\chi_{1}-\chi_{0}\right)^{2} \chi_{0}}{\chi_{1}-\chi_{0}}\right] .
\end{aligned}
$$

Accordingly, the total redshift factors are

$$
\begin{aligned}
1+z_{\mathrm{BA}}= & {\left[1+H_{0}^{\mathrm{II}}\left(2 a_{0} \chi_{0} / c\right)\right] } \\
& \times\left[1-\frac{1}{c}\left(H_{0}^{\mathrm{I}}-H_{0}^{\mathrm{II}}\right) a_{0}\left(\chi_{1}-\chi_{0}\right)\right] \\
& \times\left[1+\psi_{\mathrm{BA}}\right] \\
= & 1-\frac{a_{0}}{c}\left(H_{0}^{\mathrm{I}}-H_{0}^{\mathrm{II}}\right) \chi_{1} \\
& +\frac{a_{0}}{c}\left(H_{0}^{\mathrm{I}}+H_{0}^{\mathrm{II}}\right) \chi_{0}+\psi_{\mathrm{BA}} \\
& -\left(\frac{a_{0}}{c}\right)^{2} H_{0}^{\mathrm{II}}\left(H_{0}^{\mathrm{I}}-H_{0}^{\mathrm{II}}\right) \chi_{0}\left(\chi_{1}-\chi_{0}\right)+\ldots, \\
1+z_{\mathrm{DE}}= & {\left[1+H_{0}^{\mathrm{I}}\left(2 a_{0} \chi_{0} / c\right)\right.} \\
& \times\left[1-\frac{1}{c}\left(H_{0}^{\mathrm{I}}-H_{0}^{\mathrm{II}}\right) a_{0}\left(\chi_{1}+\chi_{0}\right)\right] \\
& \times\left[1+\psi_{\mathrm{DE}}\right] \\
= & 1-\frac{a_{0}}{c}\left(H_{0}^{\mathrm{I}}-H_{0}^{\mathrm{II}}\right) \chi_{1} \\
& +\frac{a_{0}}{c}\left(H_{0}^{\mathrm{I}}+H_{0}^{\mathrm{II}}\right) \chi_{0}+\psi_{\mathrm{DE}} \\
& -\left(\frac{a_{0}}{c}\right)^{2} H_{0}^{\mathrm{I}}\left(H_{0}^{\mathrm{I}}-H_{0}^{\mathrm{II}}\right) \chi_{0}\left(\chi_{1}+\chi_{0}\right)+\ldots .
\end{aligned}
$$

The difference of these factors reduces to

$$
\begin{aligned}
z_{\mathrm{rec}}(0)-z_{\mathrm{rec}}(\pi) \simeq & z_{\mathrm{BA}}-z_{\mathrm{DE}} \\
\simeq & \psi_{\mathrm{BA}}-\psi_{\mathrm{DE}}+\left(\frac{a_{0}}{c}\right)^{2}\left(H_{0}^{\mathrm{I}}-H_{0}^{\mathrm{II}}\right) \chi_{0} \\
& \times\left[\left(H_{0}^{\mathrm{I}}+H_{0}^{\mathrm{II}}\right) \chi_{0}+\left(H_{0}^{\mathrm{I}}-H_{0}^{\mathrm{II}}\right) \chi_{1}\right] .
\end{aligned}
$$

That is, the main terms in $\left(1+z_{\mathrm{BA}}\right)$ and $\left(1+z_{\mathrm{DE}}\right)$ cancel. The ratio $\mathscr{R}$ of $\left[z_{\text {rec }}(0)-z_{\text {rec }}(\pi)\right]$ to the relative velocity between $\mathrm{V}^{\mathrm{I}}$ and $\mathrm{V}^{\mathrm{II}}$ is

$$
\begin{aligned}
\mathscr{R} \equiv & \frac{z_{\mathrm{rec}}(0)-z_{\mathrm{rec}}(\pi)}{\left(H_{0}^{\mathrm{I}}-H_{0}^{\mathrm{II}}\right) a_{0} \chi_{0} / c} \simeq \frac{3}{4}\left[\Omega_{0}^{\mathrm{II}}\left(H_{0}^{\mathrm{II}}\right)^{2}\left(\chi_{1}+\chi_{0}\right)\right. \\
& \left.-\Omega_{0}^{\mathrm{I}}\left(H_{0}^{\mathrm{I}}\right)^{2}\left(\chi_{1}-\chi_{0}\right)\right] /\left(H_{0}^{\mathrm{I}}-H_{0}^{\mathrm{II}}\right) \\
& +2\left(H_{0}^{\mathrm{I}}+H_{0}^{\mathrm{II}}\right) \chi_{0} / c+2\left(H_{0}^{\mathrm{I}}-H_{0}^{\mathrm{II}}\right) \chi_{1} / c,
\end{aligned}
$$

where we used equation (9) for $\rho_{0}^{j}$. This ratio is the counterpart of the ratio of the velocity $v_{d}$ (corresponding to the dipole moment) to the relative (peculiar) velocity $v_{p}$, and these two ratios have comparable values.

\section{CONSISTENCY WITH THE OBSERVED LARGE-SCALE BULK FLOWS}

As was shown in $\S 2.2$, the bulk velocities in all positions within the region I are equal in the present models, so that the relative velocity $\left(v_{\mathrm{LG}}\right)$ of LG to the cluster frame is only the peculiar velocity of LG caused by GA and the nearby superclusters. Moreover, as was shown in $\S 3.3$, the dipole velocity $v_{d}$ corresponding to the bulk velocity $v_{p}$ is $\sim 0.1 v_{p}$, and so the total dipole velocity $v_{\mathrm{td}}$ of LG to CMB is $v_{\mathrm{td}}=$ $v_{\mathrm{LG}}+v_{d}\left(\sim 0.1 v_{p}\right) \cong v_{\mathrm{LG}}$.

These conclusions are consistent as follows: (1) the observed velocities $\left(v_{\mathrm{LG}}\right)$ of LG with respect to the cluster frames by Giovanelli et al. (1998), Dale et al. (1999), and Riess et al. (1997), which are nearly equal to the total dipole velocity $\left(v_{\mathrm{td}}\right)$ with respect to the CMB, and (2) the bulk velocities $\left(v_{p}\right)$ in the Streaming Motions of Abell Clusters observation (Hudson et al. 1999, 1997) and the LP10k observation (Willick 1999) are $\sim 700 \mathrm{~km} \mathrm{~s}^{-1}$ in the nearly same directions.

According to Dale et al. (1999), we have $v_{\mathrm{LG}}=565 \pm 113$ $\mathrm{km} \mathrm{s}^{-1}$ and $(l, b)=\left(267^{\circ}, 26^{\circ}\right) \pm 10^{\circ}$. On the other hand, $v_{\mathrm{td}}=627 \pm 22 \mathrm{~km} \mathrm{~s}^{-1}$ and $(l, b)=\left(276^{\circ}, 30^{\circ}\right)$ (Kogut et al. 1993). Since both directions are nearly equal, the velocity difference is about $60 \mathrm{~km} \mathrm{~s}^{-1}$. This value is comparable with $v_{d}\left(\sim 0.1 v_{p}\right)$, in the consistent manner with the result in the present models.

However, they are inconsistent with the observations of Lauer \& Postman (1994), Postman \& Lauer (1995), and Colless (1995), in which $v_{t d}$ and $v_{\mathrm{LG}}$ are in quite different directions. At present, these observations seem to have been ruled out (cf. Proceedings of the International Conference on Cosmic Flows; Courteau et al. 1999).

\section{CONCLUDING REMARKS}

In this paper we considered the behaviors of galaxies and light rays in spherically symmetric inhomogeneous models consisting of inner and outer homogeneous regions $\mathrm{V}^{\mathrm{I}}$ and $\mathrm{V}^{\mathrm{II}}$ with $\Omega_{0}^{\mathrm{I}}$ and $\Omega_{0}^{\mathrm{II}}\left(>\Omega_{0}^{\mathrm{I}}\right)$ and $H_{0}^{\mathrm{I}}$ and $H_{0}^{\mathrm{II}}\left(<H_{0}^{\mathrm{I}}\right)$, respectively, connected by a single shell. In Appendixes A and B we treat also the models with double shells and an intermediate self-similar region. It was shown that when we observe the motion of galaxies at the point $\mathrm{O}$ deviated from the center $\mathrm{C}$ in $\mathrm{V}^{\mathrm{I}}$, a constant peculiar velocity component $v_{p}$ appears in the direction from the center to the observer $(\mathrm{C} \rightarrow \mathrm{O})$. Moreover, it was shown that the velocity $v_{d}$ corresponding to the dipole anisotropy of CMB radiation is by a factor $\approx 10$ small compared with $v_{p}$. This result may fit for the observed situation of the cosmic flow of cluster galaxies, when the scale of the inner region and the distance $\mathrm{CO}$ are about 200 and $40\left(h^{\mathrm{I}}\right)^{-1} \mathrm{Mpc}$, respectively, and when 
$\left(\Omega_{0}^{\mathrm{II}}-\Omega_{0}^{\mathrm{I}}\right)$ is $\approx 0.5$ and $\left(H_{0}^{\mathrm{I}}-H_{0}^{\mathrm{II}}\right)$ is $\approx 18 h^{\mathrm{I}} \mathrm{km}^{-1} \mathrm{~s}^{-1} \mathrm{Mpc}$ (or $h^{\mathrm{II}} / h^{\mathrm{I}} \approx 0.82$ ), respectively. This difference of the Hubble parameters may be consistent with their recent values due to nearby and remote observations, because short- and long-distance scales give the Hubble constant of $H_{0} \geq 70$ and $H_{0} \sim 55$, respectively (Branch 1998; Freedman 1997; Sandage \& Tammann 1997; Blandford \& Kundić 1997). A model with multishells in the region of $100 \sim 300 \mathrm{Mpc}$ in which the parameters change stepwise will be better to reproduce the observed distribution of the Hubble constant.

It was shown that the present models are consistent with the current observations of large-scale flows (Giovanelli et al. 1998; Dale et al. 1999; Riess et al. 1997; Hudson et al. 1999, 1997; Willick 1999), but inconsistent with other obser- vations (Lauer \& Postman 1994; Postman \& Lauer 1995; Colless 1995), which may have been ruled out.

It is interesting and important to study the influences of the above inhomogeneity on the cosmological observations such as the magnitude-redshift relation of $\mathrm{SNe} \mathrm{Ia,} \mathrm{the}$ number count of galaxies, the time delay for lensed QSOs, and so on. They will be quantitatively analyzed and shown in the near future.

In this paper the motion of the LG due to the GA and superclusters in similar distances was not treated, while spherical matter distributions on such scales were analyzed by Humphreys et al. (1997). But the approximation of spherical symmetry may not be accurate because of their small-scale matter distribution.

\section{APPENDIX A}

\section{MODELS WITH DOUBLE SHELLS}

The spacetime is divided into three homogeneous regions $\mathrm{V}^{\mathrm{I}}, \mathrm{V}^{\mathrm{II}}$, and $\mathrm{V}^{\mathrm{III}}$, as shown in Figure 5. The cosmological parameters in $\mathrm{V}^{j}$ are shown as $\Omega_{0}^{j}, \lambda_{0}^{j}$, and so on, where $j=\mathrm{I}$, II, III (see Fig. 6).

Equations (1)-(3) and (9)-(15) hold in region III as well as in regions I and II. The junction condition at the second boundary between $\mathrm{V}^{\mathrm{II}}$ and $\mathrm{V}^{\mathrm{III}}$ have similar expressions to those at the first boundary:

$$
\begin{gathered}
R \equiv a^{\mathrm{II}} f^{\mathrm{II}}=a^{\mathrm{III}} f^{\mathrm{III}}, \\
\gamma^{\mathrm{III}} d\left(4 \pi R^{2} \sigma\right) / d t^{\mathrm{III}}=\left[4 \pi R^{2} \gamma^{2} v \rho\right]^{\mathrm{III}, \mathrm{II}}, \\
d\left(\gamma^{\mathrm{III}} v^{\mathrm{III}}\right) / d t^{\mathrm{III}}=-\gamma^{\mathrm{III}} v^{\mathrm{III}} H^{\mathrm{III}}+2 \pi G \sigma-\left[\gamma^{2} v^{2} \rho\right]^{\mathrm{III}} / \sigma, \\
d t^{\mathrm{II}} / d t^{\mathrm{III}}=\gamma^{\mathrm{II}} / \gamma^{\mathrm{III}}, \\
\gamma^{\mathrm{II}}\left(f^{\prime \text { II }} v^{\mathrm{II}}+H^{\mathrm{II}} R\right)=\gamma^{\mathrm{III}}\left(f^{\prime \text { III }} v^{\mathrm{III}}+H^{\mathrm{III}} R\right), \\
{\left[\gamma\left(f^{\prime}+v H R\right)\right]^{\mathrm{III}, \mathrm{II}}=-4 \pi G \sigma R,}
\end{gathered}
$$

where $[\Phi]^{\mathrm{III}, \mathrm{II}} \equiv \Phi^{\mathrm{III}}-\Phi^{\mathrm{II}}$.

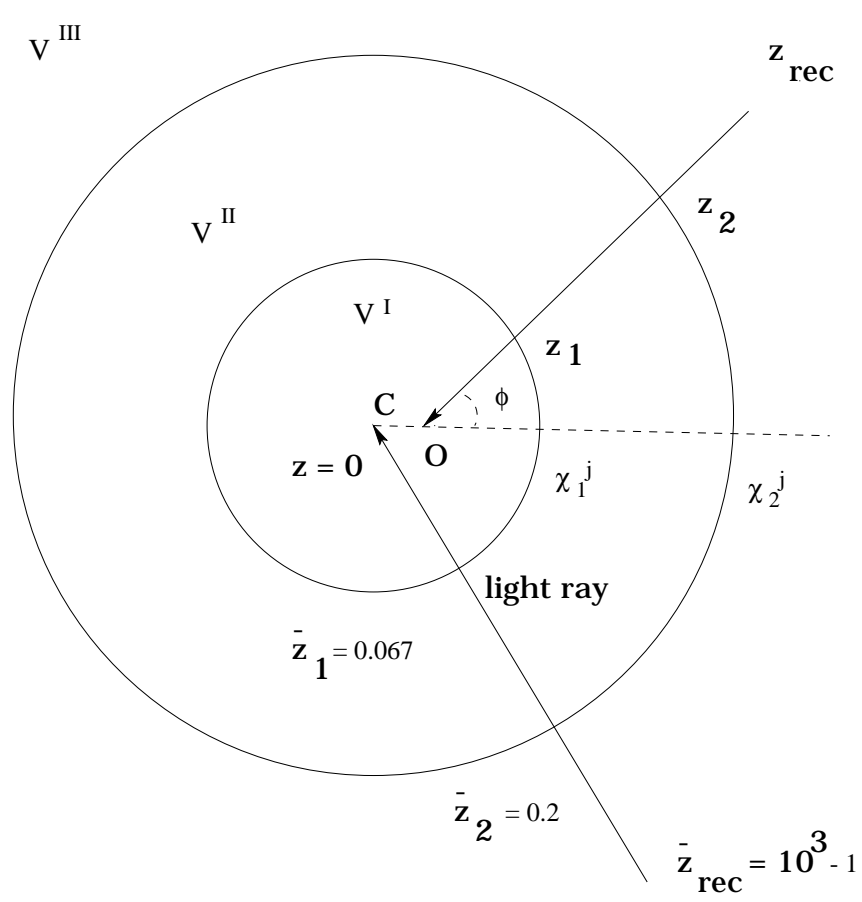

FIG. 5.- Model with double shells 


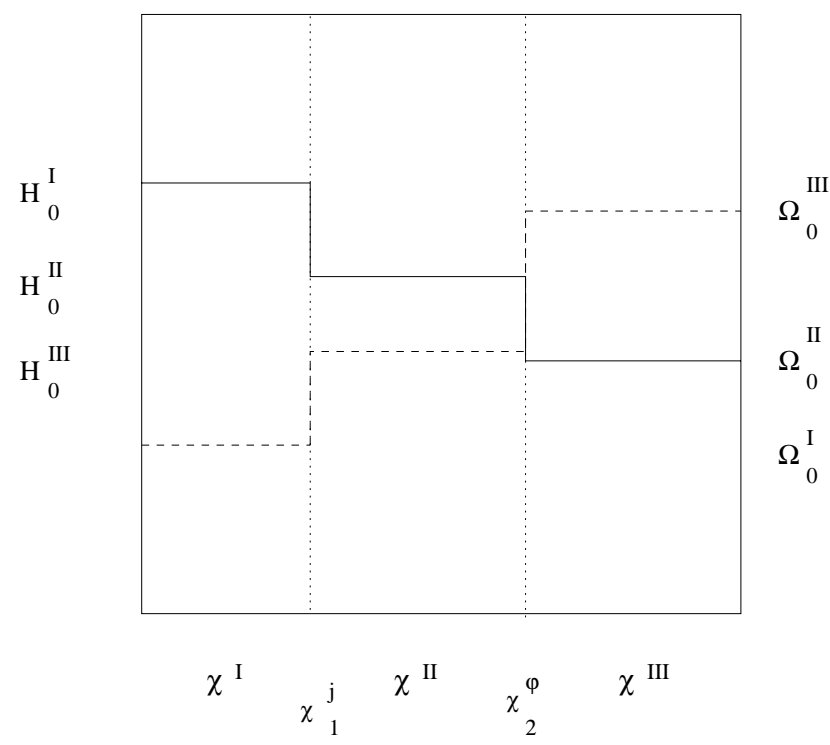

FIG. 6.-Schematic diagram of Hubble and density parameters in a model with double shells. Solid and dotted lines denote $H_{0}$ and $\Omega_{0}$, respectively.

Similar to equations (20) and (21), moreover, we have

$$
\begin{gathered}
\frac{\rho_{0}^{\mathrm{III}}}{\rho_{0}^{\mathrm{II}}}=\left(\frac{y_{i}^{\mathrm{III}}}{y_{i}^{\mathrm{II}}}\right)^{3}\left(1+\epsilon y_{i}^{\mathrm{II}}\right), \\
\left.\left(\frac{H_{0}^{\mathrm{III}}}{H_{0}^{\mathrm{II}}}\right)^{2}=\left(\frac{y_{i}^{\mathrm{III}}}{y_{i}^{\mathrm{II}}}\right)^{3}+\lambda_{0}^{\mathrm{II}}\left[1-\left(\frac{y_{i}^{\mathrm{III}}}{y_{i}^{\mathrm{II}}}\right)^{2}\right]+\frac{y_{i}^{\mathrm{III}}}{y_{i}^{\mathrm{II}}}\right)^{3}\left[1-(1+\epsilon) \frac{y_{i}^{\mathrm{II}}}{y_{i}^{\mathrm{III}}}\right] \Omega_{0}^{\mathrm{II}},
\end{gathered}
$$

where $\epsilon \approx 1$.

As for light rays, the equations in $\mathrm{V}^{\mathrm{I}}$ and $\mathrm{V}^{\mathrm{II}}$ are same as those in $\S 3$, and equations in $\mathrm{V}^{\mathrm{III}}$ are common with those in $\mathrm{V}^{\mathrm{II}}$. At the second boundary $\left(\eta=\eta_{2}, \chi=\chi_{2}\right)$, we have

$$
\begin{aligned}
\left(\frac{a_{0}}{a_{2}}\right)^{\mathrm{II}} w_{2}^{\mathrm{II}} & =\left(\frac{a_{0}}{a_{1}}\right)^{\mathrm{III}} w_{2}^{\mathrm{III}}, \\
d^{\mathrm{II}} & =d^{\mathrm{III}},
\end{aligned}
$$

where $a_{2}=a\left(\eta_{2}\right)$.

Under the assumption that the shells are comoving (i.e., $v^{\mathrm{I}}=v^{\mathrm{II}}=v^{\mathrm{III}}=0$ ), we obtain the following redshift formulas:

$$
1+z_{1}^{\mathrm{I}}=\frac{1}{y^{\mathrm{I}}}\left(\eta_{1}^{\mathrm{I}}\right)
$$

and in $\mathrm{V}^{\mathrm{II}}$ we have

$$
\begin{gathered}
\frac{1+z_{2}^{\mathrm{II}}}{1+z_{1}^{\mathrm{II}}}=\frac{y^{\mathrm{II}}\left(\eta_{1}^{\mathrm{II}}\right)}{y^{\mathrm{II}}\left(\eta_{2}^{\text {III }}\right)}, \\
\frac{1+z_{\mathrm{rec}}^{\mathrm{III}}}{1+z_{2}^{\mathrm{III}}}=\frac{y^{\mathrm{III}}\left(\eta_{2}^{\mathrm{III}}\right)}{y^{\mathrm{III}}\left(\eta_{\mathrm{rec}}^{\mathrm{III}}\right)} .
\end{gathered}
$$

From equations (6) and (A4) we have two equations: $z_{1}^{\mathrm{I}}=z_{1}^{\mathrm{II}}$ and $z_{2}^{\mathrm{II}}=z_{2}^{\mathrm{III}}$. Moreover, $\left(\eta_{1}^{\mathrm{II}}, \chi_{1}^{\mathrm{II}}\right)$ are related to $\left(\eta_{1}^{\mathrm{I}}, \chi_{1}^{\mathrm{I}}\right)$ using

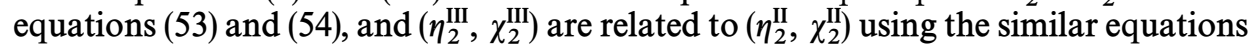

$$
\begin{aligned}
& a_{0}^{\text {II }} y^{\text {II }}\left(\eta_{2}^{\text {III }}\right) \sinh \chi_{2}^{\text {II }}=a_{0}^{\text {III }} y^{\text {III }}\left(\eta_{2}^{\text {III }}\right) \sinh \chi_{2}^{\text {III }}, \\
& a_{0}^{\mathrm{II}} \int_{0}^{\eta_{2} \mathrm{II}} y^{\mathrm{II}}\left(\eta^{\mathrm{II}}\right) d \eta^{\mathrm{II}}=a_{0}^{\mathrm{III}} \int_{0}^{\eta_{2} \mathrm{III}} y^{\mathrm{III}}\left(\eta^{\mathrm{III}}\right) d \eta^{\mathrm{III}} .
\end{aligned}
$$

First we consider the virtual observer in the center $(\chi=0)$. Then we have

$$
1+\bar{z}_{1}^{\mathrm{I}}=\frac{1}{y^{\mathrm{I}}\left(\bar{\eta}_{1}^{\mathrm{I}}\right)}
$$




$$
\begin{gathered}
\frac{1+\bar{z}_{2}^{\mathrm{II}}}{1+\bar{z}_{1}^{\mathrm{II}}}=\frac{y^{\mathrm{II}}\left(\bar{\eta}_{1}\right)^{\mathrm{II}}}{y^{\mathrm{II}}\left(\bar{\eta}_{2}\right)^{\mathrm{II}}}, \\
\frac{1+\bar{z}_{\mathrm{rec}}^{\mathrm{III}}}{1+\bar{z}_{2}^{\mathrm{III}}}=\frac{y^{\mathrm{III}}\left(\bar{\eta}_{2}\right)^{\mathrm{III}}}{y^{\mathrm{III}}\left(\bar{\eta}_{\mathrm{rec}}\right)^{\mathrm{III}}},
\end{gathered}
$$

where $\bar{\eta}_{0}^{\mathrm{I}}-\bar{\eta}_{1}^{\mathrm{I}}=\chi_{1}^{\mathrm{I}}$ and $\bar{\eta}_{2}^{\mathrm{II}}-\bar{\eta}_{1}^{\mathrm{II}}=\chi_{2}^{\mathrm{II}}-\chi_{1}^{\mathrm{II}}$. Accordingly, we can determine $\chi_{1}^{\mathrm{I}}, \chi_{2}^{\mathrm{II}}$, and $\bar{\eta}_{\text {rec }}^{\mathrm{III}}$ by specifying his redshifts $\bar{z}_{1}^{\mathrm{I}}, \bar{z}_{2}^{\mathrm{II}}$, and $\bar{z}_{\text {rec }}^{\text {III }}\left(=10^{3}-1\right)$, as in $\S 3$.

For an observer at $\mathrm{O}$ (with $\eta=\eta_{0}$ and $\chi=\chi_{0}$ ), we obtain

$$
1+z_{\mathrm{rec}}^{\mathrm{III}}=\frac{y^{\mathrm{II}}\left(\eta_{1}^{\mathrm{II}}\right) y^{\mathrm{III}}\left(\eta_{2}^{\mathrm{III}}\right)}{y^{\mathrm{I}}\left(\eta_{1}^{\mathrm{I}}\right) y^{\mathrm{II}}\left(\eta_{2}^{\mathrm{II}}\right) y^{\mathrm{III}}\left(\eta_{\mathrm{rec}}^{\mathrm{III}}\right)},
$$

where $\eta_{1}^{\mathrm{II}}, \eta_{2}^{\mathrm{II}}, \eta_{2}^{\mathrm{III}}$, and $\eta_{\text {rec }}^{\mathrm{III}}$ depend on the angle $\phi$, contrary to those in equation (A18). When we fix $\bar{z}_{1}^{\mathrm{I}}, \bar{z}_{2}^{\mathrm{II}}$, and $\bar{z}_{\text {rec }}^{\mathrm{III}}$ and determine $\chi_{1}^{\mathrm{I}}, \chi_{2}^{\mathrm{II}}$, and $\bar{\eta}_{\mathrm{rec}}^{\mathrm{III}}$, the final redshift $z_{\mathrm{rec}}^{\mathrm{III}}$ can be obtained as a function of angle $\phi$ for $\eta_{\mathrm{rec}}^{\mathrm{III}}=\bar{\eta}_{\mathrm{rec}}^{\mathrm{III}}$. It is to be noted that $\eta_{\mathrm{rec}}^{\mathrm{III}}$ is independent of the existence of the two shells.

\section{APPENDIX B}

\section{MODELS WITH AN INTERMEDIATE SELF-SIMILAR REGION}

The line element is expressed in the form (Tomita 1995, 1996)

$$
d s^{2}=-c^{2} d t^{2}+S^{2}(t, r)\left[\frac{\left(1+r S^{\prime} / S\right)^{2}}{1-k \alpha(r) r^{2}} d r^{2}+r^{2} d \Omega^{2}\right]
$$

where

$$
\alpha(r) / \alpha_{0}=1 /\left(r_{1}\right)^{2}, 1 / r^{2}, 1 /\left(r_{2}\right)^{2}
$$

for the inner homogeneous region $\mathrm{V}^{\mathrm{I}}$, the self-similar region $\mathrm{V}^{\mathrm{II}}$, and the outer homogeneous region $\mathrm{V}^{\mathrm{III}}$, respectively, which are shown in Figure 7 (see also Fig. 8). Assuming the matter pressure free and comoving, the solutions are described using the Tolman solution. The two boundaries $r=r_{1}$ and $r_{2}$ are exactly comoving. The scale factor $S$ in the case $\Lambda=0$ is given by

$$
\begin{gathered}
S / S_{0}=\frac{\Omega_{0}}{2\left(1-\Omega_{0}\right)}(\cosh \eta-1), \\
\Omega_{0}=\left(c H_{0}^{-1} / S_{0}\right)^{3}, \quad H_{0} \equiv[(\partial S / \partial t) / S]_{0} .
\end{gathered}
$$

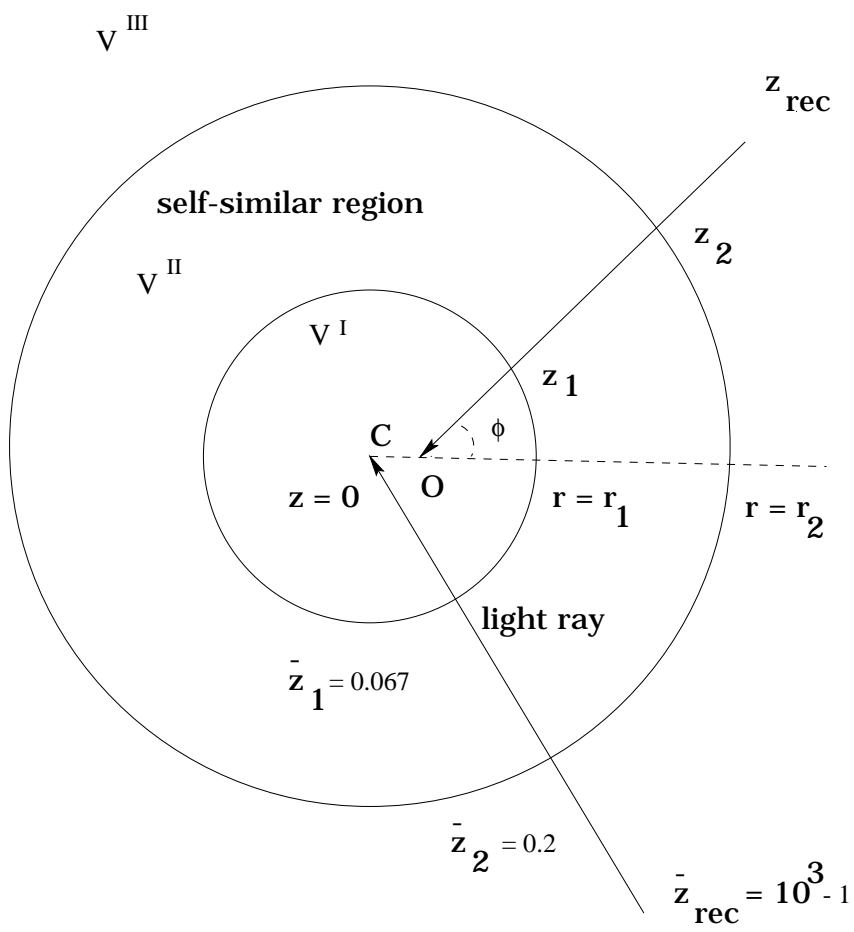

FIG. 7.-Model with the self-similar region 


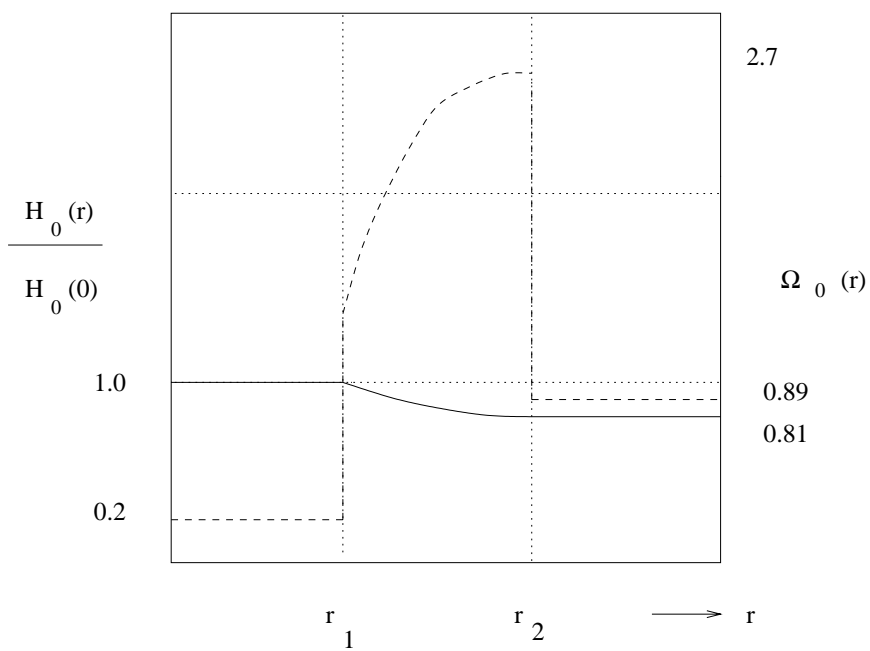

Fig. 8.-Schematic diagram of Hubble and density parameters in a model with the self-similar region. Solid and dotted lines denote $H_{0}$ and $\Omega_{0}$, respectively.

The time variable $t$ is in the inner, self-similar, and outer regions expressed as

$$
\begin{gathered}
H_{0} t=\frac{\Omega_{0}}{2\left(1-\Omega_{0}\right)^{3 / 2}}(\sinh \eta-\eta), \\
\xi \equiv \frac{c t}{r}=\frac{c H_{0}^{-1}\left(r_{1}\right)^{-1} \Omega_{0}}{2\left(1-\Omega_{0}\right)^{3 / 2}}(\sinh \eta-\eta), \\
H_{0} t=\frac{r_{2}}{r_{1}} \frac{\Omega_{0}}{\left[2\left(1-\Omega_{0}\right)^{3 / 2}\right]}(\sinh \eta-\eta),
\end{gathered}
$$

respectively. The constant $\alpha_{0}$ and $r_{1}$ are given by

$$
\begin{gathered}
\sqrt{\alpha_{0}}=\frac{4 \sqrt{1-\Omega_{0}}}{\Omega_{0}^{2}\left(1+\bar{z}_{1}\right)}\left[1-\Omega_{0}+\frac{\Omega_{0}}{2}\left(1+\bar{z}_{1}\right)+\left(\frac{\Omega_{0}}{2}-1\right) \sqrt{1+\Omega_{0} \bar{z}_{1}}\right], \\
r_{1}=\left[\left(\Omega_{0}\right)^{1 / 3} /\left(1-\Omega_{0}\right)^{1 / 2}\right] \sqrt{\alpha_{0}} .
\end{gathered}
$$

If we define the local Hubble parameter $H$ (in the transverse direction) in the $t=t_{0}$ hypersurface as

$$
H \equiv(\dot{S} / S)_{t=t_{0}}=\left[(1 / t) \xi S_{, \xi} / S\right]_{t=t_{0}},
$$

$H$ in $\mathrm{V}^{\mathrm{I}}$ is equal to $H_{0}$ and we have in $\mathrm{V}^{\mathrm{II}}$ and $\mathrm{V}^{\mathrm{I}}$

$$
H t_{0}=\frac{\sinh \eta(\sinh \bar{\eta}-\bar{\eta})}{(\cosh \bar{\eta}-1)^{2}}
$$

where $\bar{\eta} \equiv \eta_{t=t_{0}}$ satisfies

$$
H_{0} t_{0}=\left(\frac{r}{r_{1}}, \frac{r_{2}}{r_{1}}\right) \frac{\Omega_{0}}{\left[2\left(1-\Omega_{0}\right)^{3 / 2}\right]}(\sinh \bar{\eta}-\bar{\eta})
$$

for $\mathrm{V}^{\mathrm{II}}, \mathrm{V}^{\mathrm{III}}$, respectively. Present matter densities in the three regions are

$$
\rho_{0 j}=\frac{3 H_{0}^{2} \Omega_{0}}{8 \pi G}\left[1, \frac{1}{3}\left(\frac{r_{1}}{r}\right)^{2}\left(1-\xi S_{, \xi} / S\right)^{-1},\left(\frac{r_{1}}{r_{2}}\right)^{2}\right]
$$

for $j=[\mathrm{I}, \mathrm{II}, \mathrm{III}]$, respectively, and $\Omega_{0 j}$ is defined as

$$
\Omega_{0 j} \equiv \rho_{0 j}\left(t_{0}\right) /\left(\frac{3 H^{2}}{8 \pi G}\right)
$$

The behavior of light rays and redshift formulas were derived in the previous paper (Tomita 1996). If we specify the redshifts $\bar{z}_{1}, \bar{z}_{2}$, and $\bar{z}_{\text {rec }}\left(=10^{3}-1\right)$, the radii $r_{1}$ and $r_{2}$ of the two boundaries and the value of $\eta_{\text {rec }}\left(=\bar{\eta}_{\text {rec }}\right)$ are determined, and we can calculate $z_{\mathrm{rec}}$ as a function of $\phi$, which is the angle between the incident direction and the $X$-axis. 
In the following, we present additional explanations and corrections for the formulas in Appendix $\mathrm{A}$ of the previous paper (Tomita 1996). In $\mathrm{V}^{\mathrm{I}}$ and $\mathrm{V}^{\mathrm{III}}$, we have

$$
\begin{aligned}
& k^{r}= \pm\left(S_{0} / S^{2}\right)\left\{\left[1+\alpha_{0}\left(r / r_{1}\right)^{2}\right]\left[\left(w_{1}\right)^{2}-d^{2} /\left(S_{0} r\right)^{2}\right]\right\}^{1 / 2}, \\
& k^{r}=-\left(S_{0} / S^{2}\right)\left\{\left[1+\alpha_{0}\left(r / r_{2}\right)^{2}\right]\left[\left(w_{2}\right)^{2}-d^{2} /\left(S_{0} r\right)^{2}\right]\right\}^{1 / 2},
\end{aligned}
$$

respectively. The second ray in $\mathrm{V}^{\mathrm{I}}$ has

$$
h_{1}=\cosh ^{-1}\left\{\left[1+\left(\sqrt{\alpha_{0}} r_{0} / r_{1}\right)^{2}\right]^{1 / 2} / h_{0}\right\}+\left[\left(\eta-\eta_{0}\right),-\left(\eta-2 \eta_{m}+\eta_{0}\right)\right]
$$

for $\left\{\eta \geq \eta_{m}, \eta \leq \eta_{m}\right\}$, respectively. In this paper, the incoming angle is

$$
\phi=\sin ^{-1}\left(\frac{d / w_{1}}{S_{0} r_{0}}\right)\left(\equiv \phi_{1}\right), \quad \pi-\phi_{1} .
$$

Auxiliary functions $\mathrm{V}^{\mathrm{III}}$ are expressed as

$$
\begin{gathered}
M(\xi) \equiv \frac{1}{N(\xi)}\left[\left[\left(M_{0}\right)^{2}-2 d^{2} \int_{\xi 1}^{\xi} d \xi \frac{N(\xi)}{S^{3}}\left\{S S_{, \xi}+\xi\left[1+\alpha_{0}+S S_{, \xi \xi}-\left(S_{, \xi}\right)^{2}\right]\right\}\right]\right]^{1 / 2}, \\
\zeta(\xi) \equiv N(\zeta) /\left(\sqrt{1+\alpha_{0}} \xi\right) \\
\frac{M}{d}=\frac{2\left(1-\Omega_{0}\right)}{\Omega_{0} S_{0}} \frac{\cosh \eta-1}{2(1-\cosh \eta)+\eta \sinh \eta}\left(\frac{M_{0}}{d}-2 I\right) \\
\zeta=\frac{\sqrt{\alpha_{0}}}{\sqrt{1+\alpha_{0}}} \frac{2(1-\cosh \eta)+\eta \sinh \eta}{(\cosh \eta-1)(\sinh \eta-\eta)} .
\end{gathered}
$$

The constants $w_{1}$ and $w_{2}$ are connected using the junction conditions $\left(k^{0}\right)^{\mathrm{I}}=\left(k^{0}\right)^{\mathrm{II}}$ at $r=r_{1}$ and $\left(k^{0}\right)^{\mathrm{II}}=\left(k^{0}\right)^{\mathrm{III}}$ at $r=r_{2}$.

The author thanks N. Sugiyama and N. Sakai for helpful discussions and a referee for kind comments about past related works. This work was supported by Grant-in-Aid for Scientific Research (10640266) from the Ministry of Education, Science, Sports, and Culture, Japan.

\section{REFERENCES}

Blandford, R. D. \& Kundić, T. 1997, in The Extragalactic Distance Scale, ed. M. Livio, M. Donahue, \& N. Panagia (London: Cambridge Univ. Press), 60

Branch, D. 1998, ARA\&A, 36, 17

Broadhurst, T. J., Ellis, R. S., Koo, D. C., \& Szalay, A. S. 1990, Nature, 343, 726

Colless, M. 1995, AJ, 109, 1937

Courteau, S., Strauss, M., \& Willick, J., eds. 1999, ASP Conf. Ser., Cosmic Flows (San Francisco: ASP)

Dale, D. A., Giovanelli, R., \& Haynes, M. P. 1999, ApJ, 510, L11

Dressler, A., Faber, S. M., Burstein, D., Davies, R. L., Lynden-Bell, D., Terlevich, R. J., \& Wegner, G. 1987, ApJ, 313, L37

Einasto, J., et al. 1997, Nature, 385, 139

Freedman, W. L. 1997, in Critical Dialogues in Cosmology, ed. N. Turok (Singapore: World Scientific), 92

Giovanelli, R., Haynes, M. P., Freudling, W., da Costa, L. N., Salzer, J. J., \& Wegner, G. 1998, ApJ, 505, L91

Hudson, M. J., Lucey, J. R., Smith, R. J., \& Steel, J. 1997, MNRAS, 291, 488

Hudson, M. J., Smith, R. J., Lucey, J. R., Schlegel, D. J., \& Davies, R. L. 1999, ApJ, 512, L79
Humphreys, N. P., et al. 1997, ApJ, 477, 47

Israel, W. 1966, Nuovo Cimento, 44b, 1

Kogut, A., et al. 1993, ApJ, 419,

Landy, S. D., Shectman, S. A., Lin, H., Kirshner, R. P., Oemler, A., \& Tucker, D. 1996, ApJ, 456, L1

Lauer, T. R., \& Postman, M. 1994, ApJ, 425, 418

Lynden-Bell, D., Faber, S. M., Burstein, D., Davies, R. L., Dressler, A., Terlevich, R. J., \& Wegner, G. 1988, ApJ, 326, 19

Maeda, K. 1986, Gen. Relativ. Gravitation, 18, 9

Nakao, K., Gouda, N., Chiba, T., Ikeuchi, S., Nakamura, T., \& Shibata, M. 1995, ApJ, 453, 541

Postman, M. \& Lauer, T. R. 1995, ApJ, 440, 28

Riess, A. G., Davis, M., Baker, J., \& Kirshner, R. P. 1997, ApJ, 488, L1

Sakai, N., Maeda, K., \& Sato, H. 1993, Prog. Theor. Phys., 89, 1193

Sandage, A., \& Tammann, G. A. 1997, in Critical Dialogues in Cosmology, ed. N. Turok (Singapore: World Scientific), 130

Tomita, K. 1995, ApJ, 451, 541 (erratum 464, 1054 [1996])

. 1996, ApJ, 461, 507

Willick, J. A. 1999, ApJ, 522, 647

Zehavi, I., Riess, A. G., Kirshner, P., \& Dekel, A. 1998, ApJ, 503, 483 